2

3

4

5

6

\title{
ABC transporters: a riddle wrapped in a mystery inside an enigma
}

2

3

4

5

6 Peter M JONES ${ }^{1}$, Megan L O’MARA² \& Anthony M GEORGE ${ }^{1}$

7

8

9

10

11

12

13

14

15

16

17

18

19

20

21 Corresponding author

22 George, A.M (email: tony.george@uts.edu.au)

of Queensland, St Lucia, QLD 4067 AUSTRALIA

Technology Sydney, PO Box 123, Broadway, NSW 2007 AUSTRALIA

2

(1)

(1)

${ }^{2}$ Molecular Dynamics Group, School of Chemistry and Molecular Biosciences, The University

${ }^{1}$ Institute for Biotechnology of Infectious Diseases, Faculty of Science, University of 


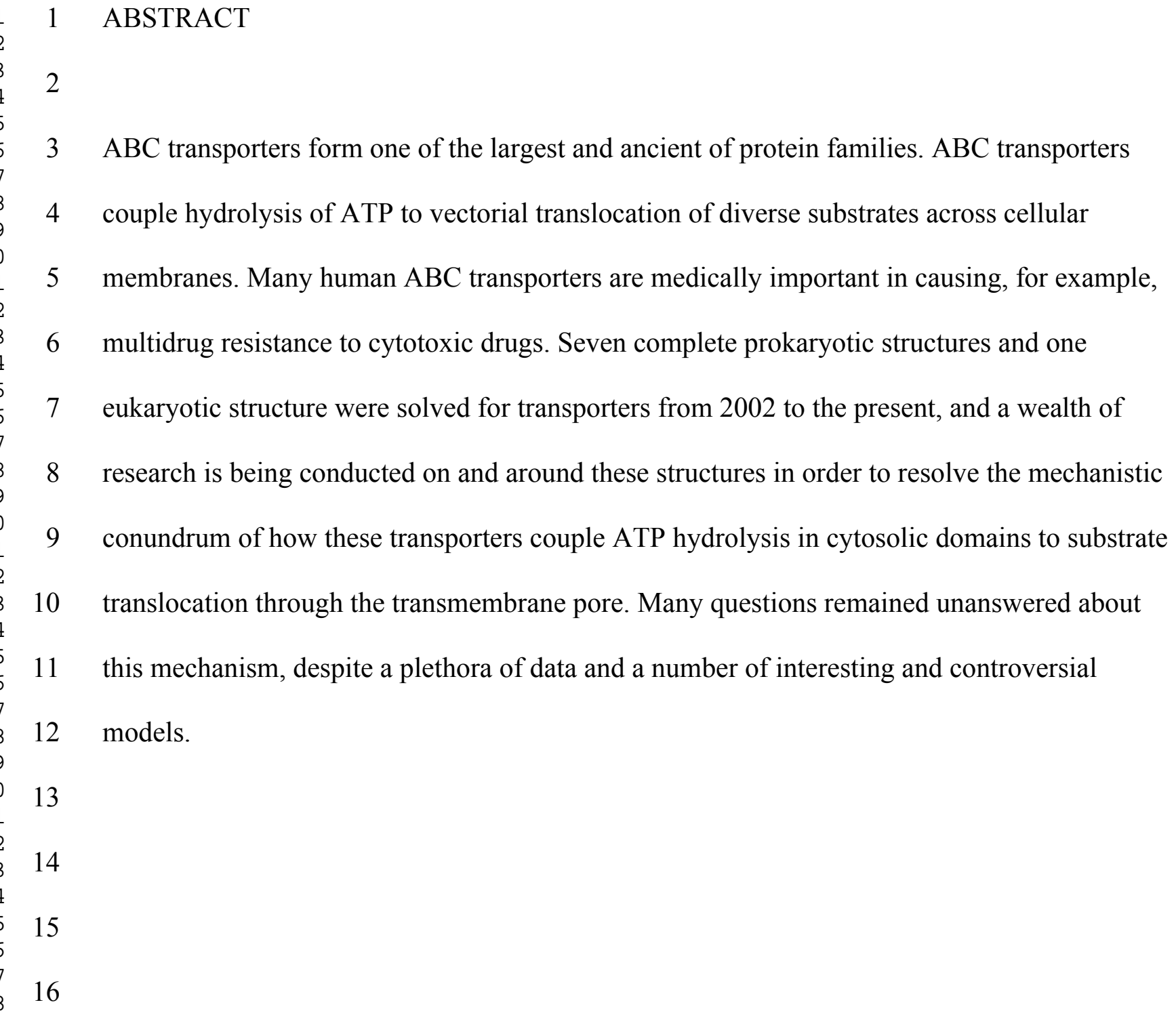




\section{INTRODUCTION}

2

\section{The $\mathrm{ABC}$ of $\mathrm{ABC}$ transporters.}

4 ATP-Binding Cassette (ABC) transporters are found in all phyla and constitute one of the

5 largest protein superfamilies [1] and [2]. A recent analysis of the full genomes from 13 diverse

6 organisms from the kingdoms, archaea, eubacteria, and eukarya identified sequences within

7 genes encoding the nucleotide-binding domains of $\mathrm{ABC}$ transporters as amongst the most

8 conserved phylogenic DNA sequences [3]. ABC transporters couple hydrolysis of ATP to

9 vectorial translocation of substrates across cellular membranes, typically against a

10 concentration gradient. Through their transport function these integral membrane proteins are

11 involved in diverse cellular processes such as maintenance of osmotic homeostasis, nutrient

12 uptake, resistance to cytotoxic drugs and antibiotics, cell division, bacterial immunity, pathogenesis and sporulation, cholesterol and lipid trafficking, cellular immune response, and developmental stem cell biology [4] and [5]. Many of the human ABC transporters are medically important, including ABCC7/CFTR that causes cystic fibrosis by any of dozens of mutations in the gene. A subclass of $\mathrm{ABC}$ transporters are associated with multidrug resistance, through the extrusion of cytotoxic agents used in chemotherapy against tumours. ABCB1/Pglycoptotein/MDR1, ABCC1/MRP1, and ABCG2/BCRP ABC transporters appear to account for nearly all of the MDR tumour cells in both humans and rodents. All of the medically important $\mathrm{ABC}$ transporters can be located at: http://www.genenames.org/genefamily/abc.html; and http://www.nutrigene.4t.com/humanabc.htm.

\section{Look at how they are built: different peas in the same pod?}

4 The architecture of $\mathrm{ABC}$ transporters comprises a conserved core structure of two transmembrane domains (TMDs) and two cytosolic ATP-binding cassettes, also known as 
nucleotide-binding domains (NBDs). The four domains may be comprised of one, two or four

2 polypeptide chains, encoded by the same or different genes, which assemble into monomers,

3 homo- or heterodimers, or tetramers. Prokaryotes harbour both importers for nutrient uptake

4 (including amino acids, sugars, metal ions) and exporters (drugs, toxins, polysaccharides,

5 lipids, proteins), and eukaryotes only exporters [6] and [7]. For example, the K12 serotype of $E$.

6 coli contains 65 experimentally verified and putative $\mathrm{ABC}$ transporters. Of the $65 \mathrm{ABC}$

7 transporters listed, 50 are $\mathrm{ABC}$ importers and the remaining 15 are exporters [8]. Bacterial

$8 \mathrm{ABC}$ importers also contain a periplasmic binding protein that captures substrate and delivers it

9 to the transporter. The TMDs, which contain cytosolic as well as membrane spanning regions,

10 form the transmembrane (TM) pore and contain the substrate binding sites, while the NBDs

11 bind and hydrolyse ATP. The cytosolic regions of the TMDs, known as the intracytoplasmic

12 loops (ICLs), form the physical interface between the TMDs and NBDs and are thought to

13 coordinate ATP binding and hydrolysis with substrate binding and translocation. Sequence

14 analysis indicates that the consensus configuration of the TMDs is of two sets of six

15 hydrophobic TM spans, though there are notable exceptions among importers, with some

16 transporters having ten or more while others have fewer than six. The TMDs are thus not well 17 conserved in length or sequence, probably reflecting their role in binding diverse substrates. In 18 a functional $\mathrm{ABC}$ transporter, two TMDs form a selectively permeable pore or conduit through

19 the membrane. At any point in time, the TMDs are "gated" so that they are closed to one side 20 of the membrane, preventing passive diffusion of substrates.

22 Each NBD is roughly an L-shape with two lobes comprising three subdomains. The larger 23 Lobe I includes a RecA- and $\mathrm{F}_{1}$-ATPase-like ATP-binding core subdomain [9] (Figure 1, blue), 24 containing the Walker A (GXXGXGKS/T) and B $(\phi \phi \phi \phi D E)$ motifs, where ' $\phi$ ' is any aliphatic 25 residue [10]. Lobe I also contains the ABC $\beta$-subdomain (Figure 1, green) that is peculiar to 
ABC-ATPases and which appears to play a predominately structural role [11]. Lobe II, also

2 known as the $\alpha$-helical subdomain (Figure 1, red), is attached flexibly to Lobe I and contains

3 the remotely located LSGGQ signature sequence that is unique to ABC-ATPase and defines the

4 family. In all ABC proteins, two ATP-binding cassette NBDs associate as a head-to-tail dimer,

5 with two ATPs sandwiched between the A and B motifs of one monomer and the signature

6 sequence of the other monomer [12] and [13]. This conformation has been observed in the

7 crystal structures of isolated NBD dimers, [13], [14], [15], [16], [17] and [18], and in some of

8 the complete $\mathrm{ABC}$ structures (see below), and is consistent with biochemical and sequence data

9 [19], [20], [21], [22] and [23]. The LSGGQ makes several main-chain and side-chain

10 hydrogen-bonded contacts with the ATP, is directly involved in catalysis of the ATP hydrolysis 11 reaction [24]. It is thought to play a role similar to the arginine finger observed in other P-loop 12 ATPases that extends from one domain into the active site of opposite domain [24]. In addition 13 to the three major motifs, a number of additional motifs and residues are involved in the ATP 14 binding and hydrolysis processes (see Glossary 'Road Map'). The import or export of substrate 15 across the TMDs is coupled to ATP hydrolysis in an allosteric manner, but the precise 16 mechanics of the process are still to be solved [25], [26] and [27].

\section{ABC transporter crystal structures: is seeing believing?}

The publication between 2001 and 2005 of three erroneous structures of the prokaryotic lipid A exporter MsbA [28] and [29] has highlighted the importance of rigorous assessment of x-ray crystallographic structures against data derived from all other sources [26], [30], and [31]. Herein, we adopt a circumspect approach to the whole transporter structures in attempting to address some of the outstanding issues regarding the structure and mechanism of ABC transporters. 
To date, one eukaryotic and ten prokaryotic ABC transporters have been crystallised,

2 representing seven different types of transporter, of which four of the structures are orthologs

3 of others. The seven transporter types display three apparently structurally unrelated TM folds

4 (Figure 2). One group comprises the BtuCD vitamin $\mathrm{B}_{12}$ importer from Escherichia coli [32]

5 and HI1470/71 metal-chelate importer from Haemophilus influenzae [33] that display two

6 densely packed block-like 10-helix bundles forming a narrow chamber within the membrane

7 (Figure 2a). The second group contains: ModBC molybdate/tungstate importer from

8 Archaeoglobus fulgidus [34], ModBC from Methanosarcina acetivorans [35], MetNI

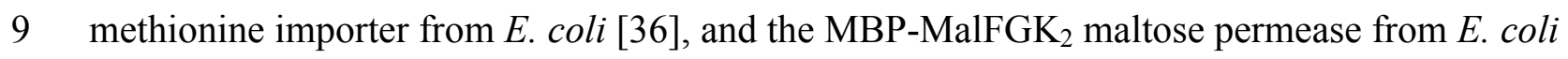

10 [37] and [38]. This group deploys 5 to 8 curved TM helices that form a relatively wide 'tepee-

11 shaped' pore (Figure 2b). The third group comprises the Sav1866 multidrug exporter from

12 Staphylococcus aureus [39], the MsbA lipid flippase exporter from E. coli [40], and the mouse 13 multidrug exporter Mdr1a [41], each displaying a $6+6 \mathrm{TM}$ helix arrangement forming two

14 large wing-like arcs (Figure 2c). Sav1866 and MsbA are close homologues of, and are

15 functionally related to, the human P-glycoprotein multidrug transporter (ABCB1 or MDR1).

16 The one structural feature common to all seven transporter types is the presence of a short 17 helix, part of the ICL from each TMD, which contacts and interacts directly with the adjacent 18 NBD, binding in a groove formed between Lobes I and II of the NBD. This helix, known as a

19 "coupling helix", is proposed to represent a part of the transmission interface whereby binding 20 and hydrolysis of ATP in the NBDs is coupled to conformational transitions in the TMDs that 21 effect solute translocation.

\section{Which transporter mechanism? Switches, tweezers, and clothes pegs.}

24 Despite the structurally unrelated nature of the TM folds, a unified model for ABC importers and exporters has been proposed and developed by comparative analysis of several full-length 
ABC structures [42]. This model is based on the allosteric model for membrane pumps

2 proposed by Jardetsky [43]. To function as a pump, a membrane protein need only meet three

3 structural conditions: (1) It must contain a cavity in the interior large enough to admit the

4 solute; (2) It must be able to assume inward-and outward-facing configurations such that the

5 cavity is alternately open to one side of the membrane; and (3) It must contain a binding site for

6 the transported species within the cavity, the affinity of which is different in the two

7 configurations. In this model, pumps for different molecules need differ only in the specificity

8 of binding sites, and the same pump molecule could be adapted to translocate more than one

9 molecular species.

11 The ABC alternating access [42] and [44] and switch [45] and [46] models predict that a 12 common mechanism of substrate translocation is utilised by both $\mathrm{ABC}$ importers and exporters 13 (Figure 3). Substrate enters the translocation pore through the TMDs, which are open to the 14 substrate delivery side and closed to the other side of the membrane, forming a partial conduit

15 across the membrane. Transport of substrate across the membrane involves structural

16 reshuffling of the TMDs, physically closing them to the substrate delivery side and opening 17 them on the opposite side of the membrane. Within the NBDs, ATP-driven closure of the NBD 18 dimer interface, in a tweezers like motion [17], causes a reduction in the distance between the

19 intracellular sides of the TMDs. This is mediated through the coupling helices $(\mathrm{CH})$, those 20 regions of the TMD intracytoplasmic loops that interact directly with the NBD (see above). The 21 change in proximity of the CHs triggers a flipping of the TMDs from an inward-facing to 22 outward-facing conformation in a clothes peg-like motion. ABC importers may now accept 23 substrates from their cognate periplasmic binding proteins (BPs), whereas ABC exporters may 24 extrude drugs or other solutes to the extracellular side of the membrane. ATP hydrolysis drives 25 the NBDs apart, flipping the TMDs from the outward-facing to inward-facing conformation to 
reset the cycle.

2

3 The Metal-chelate importers: subtle but complex.

4 The ABC importer systems BtuCD and HI1470/71 mediate the uptake of metal-chelate species

5 and are comprised of homodimeric NBDs and TMDs. Three whole transporter structures have

6 been determined for these proteins; none of the structures contain substrate or nucleotide and

7 resolutions range from $3.2 \AA$ (BtuCD) to $2.4 \AA(\mathrm{HI} 1470 / 1)$. The three structures are similar,

8 with a Root Mean Square Deviation (RMSD) for structurally equivalent residues of $2.4 \AA$

9 between the BtuCD complex and HI1470/71 [33]. Each BtuC and HI1471 TMD subunit

10 comprises 10 TM helices intricately packed in a block-like structure.

12 The E. coli BtuCD was crystallised both with and without the BP BtuF. A cavity between the 13 BtuC subunits was proposed to embody an outward-facing translocation pore. The cavity has a 14 maximum diameter of about $9 \AA[33]$ and tapers toward the extracellular mouth, such that the 15 vitamin $\mathrm{B}_{12}$ substrate (diameter $>15 \AA$ ) would require further conformational changes in the 16 TMD vestibule or cavity to enter and traverse the pore. In the BtuCD-F structure (Figure 2a), 17 one BtuC subunit is in a different conformation compared to that observed in BtuCD, resulting 18 in an asymmetric TMD:TMD conformation with no TM cavity or "pore". There are no

19 substantial structural changes of the BtuD NBD subunits in BtuCD-F when compared to 20 BtuCD, which remain in an open conformation. BtuF is bound at the extracellular face in an 21 open, ligand-releasing conformation. Both structures are proposed to represent intermediate 22 conformations in the translocation cycle [33].

24 HI1470/1 was crystallised in the absence of its cognate BP. A TM cavity formed by the 25 TMD:TMD interface is closed at the extracellular side and exhibits an inward-facing 
conformation. The cavity has a maximum diameter of $11 \AA$ [33]. Notably, both the HI1471

2 TMDs have a conformation similar to that observed in the BtuC subunit in the BtuCD-F

3 structure (above) that differs from that in the BtuCD structure. The main structural differences

4 between the two TMD conformations observed for this group were in the orientation of the

5 central pore-forming helices, particularly TM helix 5, which lines the substrate translocation

6 pore [33]. Thus, the transition from the inward to outward facing channel is due primarily to a

7 change in tilt of the pore-lining helix, TM helix 5 (Figure 4), while an asymmetric

8 conformation of the TMD subunits results in an occluded channel, two together of one

9 conformation results in an inward- or outward-facing channel.

11 Since the nucleotide-free states of BtuCD and HI1470/1 were crystallised in putative outward-

12 and inward-facing conformations respectively, no correspondence between nucleotide state and 13 transporter conformation was found [33]. The authors suggested that the substitution of the 14 native bilayer with detergent, and/or crystal lattice contacts might have shifted the equilibrium

15 between inward- and outward-facing conformations [33]. Nevertheless, the closer juxtaposition 16 of the NBDs in BtuCD relative to HI1470/1 is consistent with the idea that the outward-facing 17 conformation of the transporter corresponds to the closed, double ATP-bound state of the NBD 18 dimer (Figure 3).

20 Taken together, these structures suggested a mechanism whereby a translational shift of the 21 NBDs along the NBD dimer interface is coupled to a twisting motion of the associated 22 membrane-spanning subunits to interconvert inward- and outward-facing conformations. Thus, 23 the NBDs move in a direction perpendicular to the tweezers-type motion observed between 24 different nucleotide states of MalK, the NBD of the maltose permease [33]. More recently, 25 electron spin resonance spectroscopy was used to study the reaction cycle of BtuCD [47], based 
on the crystal structures. This study supported a model in which the NBDs remain in contact;

2 ATP binding followed by formation of the NBD sandwich dimer drives opening of the TM

3 channel at the intracellular face [32]. This is opposite to the mechanism for the other groups

4 where NBD dimer formation opens the TMDs at the extracellular face and the NBDs undergo a

5 cycle of association and dissociation. In contrast to this, a recent functional characterisation of a

6 related bacterial heme $\mathrm{ABC}$ transporter found that ATP-hydrolysis triggered release of

7 substrate at the cytoplasmic face, consistent with the models for the other structural groups

8 [48]. These latest two studies are germane to the final section of this review on NBD models

9 for binding and hydrolysis of ATP.

11 Maltose, methionine, and molybdenum transporters: symmetry and asymmetry.

12 The crystallisation of the apo (nucleotide-free) A. fulgidus ModBC-A complex revealed another 13 unique structural architecture of the TMDs. ModBC was crystallised in complex with its BP, 14 but in contrast to the asymmetric occluded pore observed in BtuCD-F, the TMDs of ModB are

15 closed to the substrate-loaded BP and extracellular side, and open to the intracellular side. The

16 homodimeric TMDs come together to form a wide inward-facing tepee (Figure $2 b$ ), and are 17 structurally symmetric. An N-terminal TM helix from each TMD swaps over to pack against 18 the outside of the helical bundle formed by the opposite TMD, but the TMD-NBD domain 19 swapping motif found in $\mathrm{ABC}$ exporters (below) is absent. A subsequent structure of $M$. 20 acetivorans ModBC, crystallized without the ModA BP, revealed a similar inward-facing 21 architecture [35]. More recently, the high-affinity methionine importer, MetNI [36], sharing the 22 same architectural fold, was crystallised in an inward-facing, nucleotide-free conformation. 23 MetNI consists of only five TM helices per TMD, lacking the N-terminal "cross-over" helix 24 observed in ModB. 
The crystal structure of the full maltose transporter complex, $\mathrm{MalFGK}_{2}$, reveals that the

2 heterodimeric TMDs contain six (MalG) and eight (MalF) helices, respectively, with each

3 having a structurally conserved core of six helices related by a pseudo-twofold symmetry. This

4 conserved core is similar to that observed for ModB and MetN, retaining the cross-over N-

5 terminal helix observed in ModB. In contrast to the ModBC-A structure, however, the MalK

6 NBDs form a closed dimer with bound ATPs, and the TMDs are open to the extracellular side

7 and closed to the intracellular side of the membrane, forming a large solvent-filled cavity that

8 reaches halfway across the predicted membrane bilayer from the periplasmic surface. The

9 maltose BP is bound to the extracellular rim of the TMDs in an open conformation, sealing the

10 pore, and the maltose substrate is bound to MalF, making no direct contacts to residues of

11 MalG [37]. In the substrate-binding site, ten residues contact the bound maltose, conferring 12 specificity and stabilizing substrate binding. Six of these residues had been identified through

13 genetic studies to severely decrease or eliminate maltose transport [37]. Interestingly, it appears

14 that some bacterial importers lack substrate binding sites in the pore, with substrate specificity

15 governed by which binding protein attaches [8] and [49].

17 The core structures of MalF, MalG, ModB and MetN are quite similar, with maximum RMSDs 18 for structurally conserved residues under $2 \AA$, less than the overall resolution of the complete

19 structures. These structures are generally regarded as establishing a conserved architecture for 20 this group, consistent with their close evolutionary relatedness [50]. A model for transport was 21 proposed in which, on ATP binding to the inward-facing conformation (Figure 2b), the tepee 22 shape will invert, alleviating a hydrophobic gate and prising open the lobes of the BP allowing 23 substrate to permeate into the pore. Comparison of the ModBC and $\mathrm{MalFGK}_{2}$ structures 24 suggests that the TMDs rotate in an essentially rigid body movement between the two 25 conformations, although modest intrasubunit conformation changes may occur. This inference 
was strongly supported most recently with the publication of the inward-facing maltose

2 permease structure [38], with comparison of the two maltose permease conformations

3 indicating that alternating access involves rigid-body rotations of the TM subdomains coupled

4 to the closure and opening of the NBD interface. Thus, in stark contrast to the metal chelate

5 transporters, the mechanism for this architectural group does not appear to involve significant

6 intrasubunit conformational changes in the TMDs, nor structural asymmetry within the TMD

7 dimer. Furthermore, the NBDs are proposed to move in a direction perpendicular to that

8 postulated for the metal chelate transporters. from their respective NBDs?

Although the proposed mechanism for this group does not involve asymmetry between the TMDs, questions of asymmetry nevertheless arise. For example, does ModB have a substrate binding site, and if so, does the substrate also bind only to one TMD, as is the case for $\mathrm{MalFGK}_{2}$ ? Given that the TMDs in the BP-less M. acetivorans ModBC structure are exactly symmetrical [35], it would appear that unless asymmetry were introduced into the ModB dimer, the asymmetric BP [51] could bind to it in one of two equivalent ways. If the outwardfacing conformation was also symmetrical, this would also be true of substrate binding to the TMDs. What role does asymmetry play, if any, in the mechanism of this architectural group? For MalFGK 2 , binding of ATP to the NBDs triggers high-affinity binding of the BP to extracellular regions of the TMDs [52]. The BP is required to close the NBD dimer interface [53] and, presumably, ATP hydrolysis requires binding of substrate to the outward-facing TMDs. For this architectural group, since the BP and the substrate must interact with the TMD subunits in an asymmetric manner, how do both TMDs exchange equivalent signals to and

\section{Multidrug exporters: crossing over to the other side.}


The TMD structure of the three ABC exporters crystallised, Sav1866 [39], [54], the revised

2 MsbA [40], and Mdr1a [41], exhibits yet another unique ABC transporter architecture (Figure

$32 \mathrm{c}, \mathrm{d})$. There are nine published structures for these ABC exporters with resolutions ranging

4 from $3.0 \AA$ for the ADP bound form of Sav1866 to $5.5 \AA$ for the nucleotide-free (apo) MsbA

5 structures, For the latter, $\mathrm{C} \alpha$ coordinates only are available. The nucleotide-bound

6 conformations of Sav1866 and MsbA are almost identical, with an RMSD of $<2.2 \AA$ between

7 the $\mathrm{C} \alpha$ positions of the monomers [40]. The structure of each half-transporter comprises six

$8 \mathrm{TM}$ helices in the TMD, followed by a canonical $\mathrm{ABC}$ transporter $\mathrm{NBD}$, covalently joined to

9 the intracellular extension of TM6. Of the six TM helices in each half-transporter, TMs 2-6 extend significantly beyond the predicted extent of the bilayer into the cytoplasm, having an average length of almost $70 \AA$. The ICLs between TM helices 2 and 3 and between TM helices 4 and 5, (ICL1 and ICL2) each form a pair of antiparallel $\alpha$-helices, connected by an 8-12 amino acid $\alpha$-helical section, $\mathrm{CH} 1$ and $\mathrm{CH} 2$, respectively. These sections of the ICLs are the most distal to the membrane and form the interface with the NBDs (Figure 2d).

Perhaps the most striking and emblematic feature of the nucleotide-bound Sav1866 and MsbA structures is that ICL2 from each TMD crosses over to contact exclusively the NBD from the opposite monomer in the dimeric arrangement, while ICL1 predominately, although not exclusively, contacts the NBD within its own protomer. Although the possibility of domain swapping involving the ICLs had been essayed earlier on the basis of modelling [55], this result was generally unexpected. Thus, ICL1 and the intracellular extension of TM6 from one protomer combine with ICL2 from the opposite protomer to form a 5-helix globular domain that is stabilised by internal hydrophobic packing and associated predominately with the NBD covalently linked to the respective TM6. The domain swapping continues through the TM regions, where TM helices 4 and 5 reach across away from one of the TMDs and form the 
majority of their inter-helical contacts with TM helices from the opposite protomer, resulting in

2 a lobular or winged appearance of the TMDs. In the nucleotide-bound state, the TMD wings

3 form a chamber, closed at the cytoplasmic side by the apposition of the two ICL domains and

4 splaying outward in a "V" shape to flank the wide extracellular opening of the chamber (Figure

5 2c). The gap between the two wings in the exporters runs approximately perpendicular with

6 respect to the NBDs to that observed for the TMDs in the structures of the importers (Figure 2).

7 Thus, the switching between inward and outward facing conformations in exporters would

8 involve completely different coupling of conformational changes in the TMDs to the putative

9 opening and closing of the NBD dimer.

11 Although the Sav1866 and revised "closed" AMP-PNP-bound MsbA structures are generally 12 considered to fit well with other experimental data, some inconsistencies warrant mention.

13 Dong et al. [56] investigated the structure of MsbA in liposomes trapped in four intermediate 14 states, including apo and AMP-PNP-bound. Notably, this study found that residues in the N15 terminal half of TM helix 6, (residues 284-296), showed very low accessibility to the aqueous 16 medium in all phases of the transport cycle examined. These accessibility data are in excellent 17 agreement with cysteine mutagenesis studies of the equivalent region (residues 331-343) in $18 \mathrm{ABCB} 1$ [57], which showed that, with the exception of residue 343, this region was

19 inaccessible to a hydrophilic reagent in all phases of the transport cycle. From the nucleotide20 bound Sav1866 and MsbA structures, the N-terminal half of TM helix 6 is expected to form 21 part of the interior wall of the TMD chamber, with its $\mathrm{N}$-terminus projecting beyond the bilayer 22 into the extracellular milieu and its C-terminal residues deeper within the TMD channel. Since 23 the outward-facing Sav1866 and MsbA are open to the cell exterior, the structures clearly 24 indicate that residues in the N-terminal half of TM6 would be accessible to the bulk solvent 25 (with a helical periodicity) at some stage of the transport cycle. 
2 Whilst cysteine cross-linking experiments using ABCB1 [58], CFTR [59], TAP [60], and

3 Yor1p [61] have provided convincing evidence that these transporters deploy a similar domain

4 swapping TMD:NBD interface to that observed in the Sav1866, results from other studies of

5 the ABCB1 TM regions do not fit quite as well. Homology models of ABCB1 based on the

6 Sav1866 structure [62] and [63] were used to assess cross-linking data for ABCB1 for residues

7 within the TM segments, indicating that while the majority of the data fit the models quite well,

8 a significant number of discrepancies remain. These may be resolved by structures of

9 alternative conformations. Finally, recent low to medium resolution EM images of ABC

10 exporters have tended to support the nucleotide-bound Sav1866 and MsbA structures [64] and

11 [65]. Nevertheless, it is also true that EM studies of ABCB1 [66], [67], [68], [69] and [70], and

12 of the related exporters human MRP1 [67] and TAP1/2 [71], consistently show that, in the nucleotide-free state, the TMDs are open to the extracellular face. This does not agree with the exporter structures in that the nucleotide-bound and apo states cannot both be open at the extracellular face (Figure 2c).

Most recently, the whole transporter structure of mouse Mdr1a P-glycoprotein, which shares 87\% sequence identity with human P-glycoprotein, was published [41]. In this nucleotide-free structure, the NBDs are separated by $\sim 30 \AA$ and the inward-facing conformation of the TMs results in a large internal cavity open to both the cytoplasm and the inner leaflet of the membrane. Structures were obtained both with and without bound substrate, but substratebinding did not alter the transporter conformation. The inward-facing structure does not allow substrate access from the outer membrane leaflet nor the extracellular space but would freely allow both lipids and substrates to enter the putative substrate binding chamber from the membrane [41]. This is consistent with data suggesting that P-glycoprotein may act like a 
hydrophobic vacuum cleaner, binding substrates directly from the inner leaflet of the membrane [72].

3

The Mdrla structure shares the domain swapping and NBD:TMD interface observed in the prokaryotic exporters. However, the inward-facing conformation of the TMDs is not achieved by a rigid body motion as in the maltose permease and related importers, nor by the subtle intrasubunit changes observed in the metal chelate importers. Rather, the transition between the inward- to outward-facing conformation is achieved by radical rearrangement of the TM helices with respect to that observed in nucleotide-bound Sav1866 and MsbA, in a manner similar to that observed in the nucleotide-free MsbA structures [40], reminiscent of a child's origami fortune teller game. In the nucleotide-free Mdr1 a conformation, the gap between the pseudo-symmetrical TM wings runs transverse with respect to the NBDs to that observed in the outward-facing nucleotide-bound conformations of Sav1866 and closed MsbA structures. It was noted that further opening of the TM cavity and concomitant movement apart of the NBDs may be required to allow some substrates access to the cavity. Notably however, the structure does not appear to resolve many of the discrepancies with cysteine cross-linking data and the correlation between the residues comprising the putative drug-binding sites and those identified by extensive biochemical studies. Curiously, the apo and drug-bound structures do not differ in conformation and the manner in which substrate binding promotes ATP binding [46] is not apparent. However, at $3.8 \AA$, the crystal structure is of relatively low resolution and only $65 \%$ of residues are located in favoured regions of the Ramachandran plot, suggesting further refinement is needed. Finally, as observed above, the closed extracellular face does not appear consistent with extensive EM data of nucleotide-free P-glycoprotein.

\section{Evolutionary considerations: all in the family?}


Prior to the publication of full length structures of $\mathrm{ABC}$ transporters, in line with evolutionary

2 theory and as predicted in the Jardetsky model [43] (above), it was generally expected that they

3 would have evolved from a common progenitor, with a conserved overall structure and

4 mechanism adapted to different substrates. Thus, the different types of TM structures observed

5 in the crystallographic studies have led to the suggestion that the TMDs of ABC transporters

6 may have several unrelated ancestors [46]. However, sequence analysis has shown a good

7 correlation between the phylogenetic classification of NBDs, TMDs and BPs [73], [74] and

8 [75], which is not supportive of this idea [76].

The evolutionary time required for the NBD to pair with a new domain and form an efficiently functioning transporter for a particular substrate would be considerably greater than that required to adapt an existing working design to a new substrate. Phylogenetic analysis of $\mathrm{ABC}$ genes concluded that the uptake function was separated from the export function early before the divergence between prokaryotes and eukaryotes and that this separation occurred once in the history of $\mathrm{ABC}$ systems [75]. Since this division is at the root of the $\mathrm{ABC}$ transporter NBD phylogenetic tree, pairing of the NBD to new domains would have occurred subsequent to this event, indicating that grafting to new domains would have had to outstrip development of an existing design. Nonetheless, since the phylogeny of ABC genes closely correlates with substrate specificity, the structural data suggest that yet more unique $\mathrm{ABC}$ transporter TMD folds may exist [44]. It will be intriguing to learn what evolutionary pressures stimulated the development of new designs, particularly considering the very diverse range of substrates that the exporter folds of P-glycoprotein and the MRPs are adapted to transport.

4 To date, the only correlation suggested between a specific property of the substrate and the TMDs is its size, since it appears that broadly the larger the substrate the longer the TMD 
sequence, and this seems reasonable since larger substrates would require a larger TM channel

2 [32]. However, in comparing for example the vitamin $\mathrm{B}_{12}$ and methionine importer TMDs, we

3 find that although BtuC (326 residues) is significantly larger than MetI (208 residues), BtuC

4 does not appear to devote the additional residues to expanding the channel relative to that

5 observed for MetI, but rather curiously folds into an intricately and densely packed 10-helix

6 bundle with a narrower channel formed at the TMD:TMD interface than that observed for

7 MetI. What attributes of the BtuC structure could conceivably make it peculiarly suited to the

8 vitamin $\mathrm{B}_{12}$ substrate? The answer to these questions may, however, be more complex than

9 simply the structure of the TMD. For example, the TMD and NBD components of the

10 prokaryotic arginine and histidine permeases are identical, consisting of a single copy of each

11 transcribed HisM and HisQ protein for the TMDs and two copies of the transcribed HisP as the

12 NBD. The functional difference between the two transporter systems arises from the presence

13 of an arginine-specific (ArgT) or histidine-specific (HisJ) BP, which delivers the import

14 substrate to the transporter complex. This "modular" expression reduces the necessity for entire

15 gene duplication and allows for greater genetic variation in bacterial $\mathrm{ABC}$ transporter genomes.

16

17 Harnessing the engine: is one as good as two?

18 The homology of ABCs in all classes and their similarity in structure strongly suggest that

19 these components of $\mathrm{ABC}$ transporters all work in the same way [49]. The whole transporter

20 structures indicate that the contacts between the NBDs and the TMDs are significantly more 21 extensive in the nucleotide-bound exporters than in the importers, with no equivalent in the 22 importers to $\mathrm{CH} 1$ (Figure 5). In exporters, $\mathrm{CH} 1$ interacts directly with regions contacting the 23 nucleotide adenine ring, acting to sequester the nucleotide and the active site from the bulk 24 solvent in the ATP-bound state. Thus, calculation of the solvent accessible surface with the 25 program VMD (Visual Molecular Dynamics) [77] reveals that, in the ATP-bound maltose 
importer structure, atoms of the pyrophosphate moiety, the conserved glutamine, and the

2 Walker B aspartate and glutamate, are all directly accessible to the bulk solvent, while in

3 Sav1866 and closed MsbA they are not.

5 Is the lack of an equivalent to $\mathrm{CH} 1$ in importers at odds with notion that the NBDs all function

6 the same way? It is very likely that in occluding the nucleotide, $\mathrm{CH} 1$ in exporters would alter

7 the dielectric and environment of the highly conserved active site and act to detect the

8 nucleotide. Interestingly, it has been shown for both $\mathrm{ABC}$ importers and exporters that

9 vanadate trapping, which mimics the pentacoordinate transition state of ATP hydrolysis, results

10 in an occluded state of the nucleotide in the active site [76] and [78]. Since this occlusion does

11 not occur in the isolated NBDs, it must require the participation of the TMDs. In the exporters,

$12 \mathrm{CH} 1$ occludes the nucleotide and sits along side $\mathrm{CH} 2$ (Figure 5), which binds in the groove

13 between Lobes I and II, as does the single $\mathrm{CH}$ in the importers. Thus, the single $\mathrm{CH}$ in

14 importers cannot occlude the nucleotide as does $\mathrm{CH} 1$ in exporters. How is the nucleotide

15 occluded in vanadate-trapped importers?

16

\section{The nucleotide-binding domains: an open and shut case?}

The most subscribed model for the functioning of the NBDs was based originally on the evidence of crystal structures, which show that in the ATP-bound state, the NBDs form a closed dimer with two ATP molecules bound at the dimer interface, while nucleotide-free structures consistently show the NBDs physically separated. Thus, in this model (Figure 6a), variously known as the 'processive clamp' [13] and [22], 'tweezers-like' [17], or 'switch' model [45] and [46], each spatially separated NBD of a dimer pair binds a molecule of ATP, followed by the formation of a closed NBD dimer and sequential hydrolysis of the two ATPs. Nucleotide hydrolysis in turn induces the NBD monomers to fully separate to allow expulsion 
of ADP and renewed ATP binding. Pi release may occur prior to NBD separation. The alternate

2 opening and closing of the NBD dimer is proposed to couple directly to the change in the

3 inward-outward orientation of the TMDs that enable substrate translocation (see above). It is

4 notable that the majority of support for the existence of the symmetrical sandwich dimer comes

5 from structural and biochemical studies in which NBD catalysis of ATP hydrolysis was

6 abrogated, either through mutation of catalytic residues, the use of non-hydrolysable ATP

7 analogues or absence of the catalytic divalent metal [79].

9 The nucleotide-free states of full transporters or dimeric NBDs (e.g. MalK) exhibit significant

10 differences, suggesting that no strict geometric constraints exist in this state [34]. Indeed, in

11 some structures (MetNI, MalK dimer, MsbA open), the LSGGQ and the opposite Walker A are

12 more than $20 \AA$ apart, across what would be a solvent filled gap in vivo, and thus the NBDs are

13 not in direct contact. Although it is proposed that ATP binding and the subsequent tight closure

14 of the NBD dimer, provides the free energy for the "power stroke" of the transport complex 15 [13], [14], [17], [22], [46], [80] and [81], it is difficult to envisage how ATP binding could 16 achieve this. The distances between the NBD monomers and their variable geometries in the 17 apo state, together with the fact that $\mathrm{MgADP}^{-}$and $\mathrm{MgATP}^{2-}$ differ by only one charge unit, 18 makes it appear unlikely that electrostatic forces could bring the monomers together at all, far 19 less in a sufficiently timely and accurate manner as to be a crucial step of a conserved protein 20 mechanism. Indeed, what could be the relevance of the apo state observed in the crystal 21 structures given that the physiological ATP concentration is 10 times greater than the Km of 22 ATP binding to the NBD [37]? In regard to these ideas, it is interesting to note that the earlier 23 version of the switch model [46] was modified recently [82] to state that the close proximity of 24 the NBDs in intact $\mathrm{ABC}$ transporters suggests that the structural differences between the open 25 and closed dimers are probably subtle rather than complete dissociation. 
2 Prior to the first crystal structures revealing the double ATP-bound NBD sandwich dimer, it

3 was regarded generally as a central tenet that the ABC transporter NBDs hydrolysed ATP

4 alternately and that the active sites were closely coupled throughout the catalytic cycle,

5 consistent with an asymmetric mechanism with respect to the NBD monomers, the so-called

6 "alternating sites" model (Figure 6b) [76]. This notion, however, although founded on classic

7 biochemical studies, has been, in the main, eschewed in the wake of the crystal structures; and

8 asymmetry and tight coupling between the active sites play only a peripheral and ill-defined

9 role in currently popular mechanistic schemes. The source of the incompatibility is in large part

10 because the structures indicate unambiguously that the NBDs must move apart in a

11 symmetrical rigid body fashion to enable the opening of the pseudo-symmetrical TMDs to the

12 cytoplasm. In this mechanism, there appears no need for the NBDs to hydrolyse ATP

13 alternately and although attempts have been made to incorporate these ideas [83] into the so

14 called "switch model", the purpose of alternating ATP hydrolysis in this context remains

15 obscure.

16 monomers to separate fully. 
11 Enzymological data from functional whole transporters support an asymmetric model for NBD

2 dimer function. EPR analysis of homomeric MsbA consistently showed the existence of

3 asymmetric environments for all spin label pairs placed in the Walker A and LSGGQ motifs,

4 and within the Q-, H- and D-loops (see Glossary) [79], [87] and [88]. Cysteine cross-linking

5 experiments using ABCB1 revealed that the trapping of ATP, or transition state analogue at

6 one active site, resulted in reduced contact between Lobe I of NBD1 and the ICLs [58],

7 consistent with opening of one active site while the other is closed. In addition, in vanadate-

8 trapped $\mathrm{ABCB} 1$, the nucleotide-bound active site is occluded while the other is empty and

9 accessible to the bulk solvent [89]. Other data support the notion that the NBDs do not

10 dissociate. Experiments with the functional maltose importer showed that the helical

11 subdomains do not move apart substantially during the catalytic cycle [90]. Finally,

12 accessibility data from functional MsbA suggested that the LSGGQ region has low solvent

13 accessibility throughout the ATP hydrolysis cycle [88] contradicting its solvent accessible

14 placement as observed in nucleotide-free whole transporter structures.

15

16

17 Concluding remarks: quo vadis?

Although significant breakthroughs in structural studies of $A B C$ transporters have continued apace since early this decade, it is probably fair to say that our clarity regarding their molecular mechanisms has not increased in direct proportion. This is due in part to the difficulty in obtaining structures of the same transporter in different stages of the transport cycle. Whilst a unified model for transport in importers and exporters was proposed to project the Jardetsky concept [43] onto $\mathrm{ABC}$ transporters [44], it was suggested recently that the metal chelate group may operate in a distinct way. For this latter group, the most recently proposed model [47] 
differs from that earlier inferred from the BtuCD, BtuCDF and HI1470/71 structures (see above) and the mechanism for this architecture awaits further elaboration.

3

4 The situation appears far clearer cut with respect to the maltose permease group, with structures 5 of the inward- [38] and outward-facing [49] maltose permease structures illustrating both states

6 of the two-phase model very well. Nevertheless, much is still required to understand how these

7 transporters work. In particular, whilst it is clear that signals between the NBDs and

8 extracellular regions must occur, how these are propagated and coordinated do not appear to be

9 revealed by the structures, nor addressed in current models. For exporters, significant questions

10 regarding their mechanism remain. Even the now well-regarded Sav1866 structure is

11 constrained conformationally by the intertwined TMD 'wings' and domain-swapped NBD

12 ICLs, prompting the authors to suggest that the two subunits are unlikely to move independently and their maximum separation during the transport cycle is therefore limited [39]. This inference disagrees with the conformations observed in the nucleotide-free MsbA and Mdr1a structures, in which the NBDs are more than $30 \AA$ apart. In addition, although the location of the substrate-binding sites appears to be revealed in the Mdrla structures, the structural basis of substrate-stimulated ATP hydrolysis remain unknown, despite the existence of nine structures and a wealth of data for $\mathrm{ABC}$ transporters.

Perhaps the central conundrum that emerges from consideration of the coupling of the NBDs to the TMDs is that the notion of a single substrate-binding site, formed by a pair of symmetrical TMDs and alternating between inward- and outward-facing conformations, is difficult to reconcile convincingly with an alternating asymmetric functioning in the NBDs. This is because it would require one hydrolysis event to alter the conformation of the substrate-binding site in one direction while the other hydrolysis event would alter it in the opposite direction, 
11 which at least for homodimeric transporters, seems unlikely if not impossible. The NBD switch

2 model is the logical consequence of the single-substrate binding site idea, but even it cannot

3 escape from notions of asymmetry because the NBD dimer has two equal substrate-binding

4 sites that cannot behave in a symmetrical manner at all times. Notably, prior to the whole

5 structures, a "two-cylinder engine" model for asymmetric functioning of the TMDs was

6 proposed for P-glycoprotein based wholly on biochemical data [91].

8 It is clear that many significant questions remain with respect to the structure and mechanism

9 of $\mathrm{ABC}$ transporters and it would appear that answers must await more structures of

10 transporters from each group in different stages of the transport cycle. We hope that the

11 questions asked and observations made in this review will help to stimulate thought and

12 discussion and thus contribute to the advancement of our understanding of these important and 13 intriguing proteins.

16 ACKNOWLEDMENTS

17 This work was supported by a Cure Cancer Australia Fellowship to P.M.J. and a University of 18 Queensland Post-doctoral fellowship to M.L.O.

21 REFERENCES

1. Higgins, C.F. (1992) ABC transporters: from microorganisms to man. Annu. Rev. Cell Biol. 8, 67-113 
2. Holland, I.B. and Blight, M.A. (1999) ABC-ATPases, adaptable energy generators fuelling transmembrane movement of a variety of molecules in organisms from bacteria to humans. J. Mol. Biol. 293, 381-399

3. Isenbarger, T.A. et al. (2008) The most conserved genome segments for life detection on Earth and other planets. Orig. Life Evol. Biosph. 38, 517-533

6. Bouige, P. et al. (2002) Phylogenetic and functional classification of ATP-binding cassette (ABC) systems. Curr. Protein Pept. Sci. 3, 541-559

4. Dean, M. et al. (2001) The human ATP-binding cassette (ABC) transporter superfamily. Genome Res. 11, 1156-1166

5. Holland, I.B., Cole, S.P.C., Kuchler, K. and Higgins, C.F. (Eds.) (2003) ABC proteins: from bacteria to man. Academic Press, UK

7. Davidson, A.L. et al. (2008) Structure, function, and evolution of bacterial ATP-binding cassette systems. Microbiol. Mol. Biol. Rev. 72, 317-364

8. Moussatova, A. et al. (2008) ATP-binding cassette transporters in Escherichia coli. Biochim. Biophys. Acta 1718, 1757-1771

9. Vetter, I.R. and Wittinghofer, A. (1999) Nucleoside triphosphate-binding proteins: different scaffolds to achieve phosphoryl transfer. Q. Rev. Biophys. 32, 1-56 
10. Walker, J.E. et al. (1982) Distantly related sequences in the $\alpha$ - and $\beta$-subunits of ATP synthase, myosin, kinases and other ATP-requiring enzymes and a common nucleotide binding fold. $E M B O J .1,945-951$

5 11. Karpowich, N. et al. (2001) Crystal structures of the MJ1267 ATP binding cassette reveal an induced-fit effect at the ATPase active site of an ABC transporter. Structure 9, 571-586

13. Smith, P.C. et al. (2002) ATP binding to the motor domain from an ABC transporter drives formation of a nucleotide sandwich dimer. Mol. Cell 10, 139-149

12. Jones, P.M. and George, A.M. (1999) Subunit interactions in ABC transporters: towards a functional architecture. FEMS Microbiol. Lett. 179, 187-202

14. Hopfner, K-P. et al. (2000) Structural biology of Rad50 ATPase: ATP-driven conformational control in DNA double-strand break repair and the ABC-ATPase superfamily. Cell 101, 789-800

15. Lamers M.H. et al. (2000) The crystal structure of mismatch repair protein MutS binding to a G:T mismatch. Nature $407,711-717$

16. Obmolova, G. et al. (2000) Crystal structures of mismatch repair protein MutS and its complex with a substrate DNA. Nature 407, 703-710

17. Chen, J. et al. (2003). A tweezers-like motion of the ATP-binding cassette dimer in an ABC transport cycle. Mol. Cell 12, 651-661 
18. Pakotiprapha, D. et al. (2008) Crystal structure of Bacillus stearothermophilus UvrA provides insight into ATP-modulated dimerization, UvrB interaction, and DNA binding. Mol. Cell. 29, 122-133

19. Chen, J. et al. (2001) Trapping the transition state of an ATP-binding cassette transporter: Evidence for a concerted mechanism of maltose transport. Proc. Natl. Acad. Sci. USA 98, $1525-1530$

20. Loo, T.W. et al. (2002) The "LSGGQ" motif in each nucleotide-binding domain in human P-glycoprotein is adjacent to the opposing walker A sequence. J. Biol. Chem. 277, 41303-
21. Moody, J.E. et al. (2002) Cooperative, ATP-dependent association of the nucleotide binding cassettes during the catalytic cycle of ATP-binding cassette transporters. J. Biol. Chem. 277, 21111-21114

22. Janas, E. et al. (2003) The ATP hydrolysis cycle of the nucleotide-binding domain of the mitochondrial ATP-binding cassette transporter Mdl1p. J. Biol. Chem. 278, 26862-26869

23. Zaitseva, J. et al. (2006) A structural analysis of asymmetry required for catalytic activity of an ABC ATPase domain dimer. EMBO J. 24, 1901-1910

24. Ye, J. et al. (2004) RecA-like motor ATPases-lessons from structures. Biochim. Biophys. Acta $1659,1-18$ 
11

25. Schmitt, L. and Tampe, R. (2002) Structure and mechanism of ABC transporters. Curr. Opin. Struct. Biol. 12, 754-760

26. Jones, P.M. and George, A.M. (2004) The ABC transporter structure and mechanism: perspectives on recent research. Cell. Mol. Life Sci. 61, 682-699

27. Procko, E. et al. (2009) The mechanism of ABC transporters: general lessons from structural and functional studies of an antigenic peptide transporter. FASEB J. 23, 1287 1302

28. Petsko, G.A. (2007) And the second shall be first. Genome Biol. 8, 103

29. Jeffrey, P.D. (2009) Analysis of errors in the structure determination of MsbA. Acta Crystallogr. D. Biol. Crystallogr. 65, 193-199

30. Campbell, J.D. et al. (2003) Extending the structure of an ABC transporter to atomic resolution: modelling and simulation studies of MsbA. Biochemistry 42, 3666-3673

31. Stenham, D.R. et al. (2003). An atomic detail model for the human ATP binding cassette transporter P-glycoprotein derived from disulfide cross-linking and homology modelling. FASEB J. 17, 2287-2289

32. Locher, K.P. et al. (2002) The E. coli BtuCD structure: a framework for ABC transporter architecture and mechanism. Science 296, 1091-1098 
33. Pinkett, H.W. et al. (2007) An inward-facing conformation of a putative metal-chelatetype ABC transporter. Science 315, 373-377

34. Hollenstein, K. et al. (2007) Structure of an ABC transporter in complex with its binding protein. Nature 446, 213-216

35. Gerber, S. et al. (2008) Structural basis of trans-inhibition in a molybdate/tungstate ABC transporter. Science 231, 246-250

36. Kadaba, N.S. et al. (2008) The high-affinity E. coli methionine ABC transporter: structure and allosteric regulation. Science 321, 250-253

37. Oldham, D.M. et al. (2007) Crystal structure of the catalytic intermediate of the maltose transporter. Nature 450, 515-521

38. Khare, D. et al. (2009) Alternating access in maltose transporter mediated by rigid-body rotations. Mol. Cell 33, 528-536

39. Dawson, R.J.P. and Locher, K.P. (2006) Structure of a bacterial multidrug ABC transporter. Nature 443, 180-185

40. Ward, A. et al. (2007) Flexibility in the ABC transporter MsbA: alternating access with a twist. Proc. Natl. Acad. Sci. USA 104, 19005-19010 
41. Aller, S.G. et al. (2009) Structure of P-glycoprotein reveals a molecular basis for polyspecific drug binding. Science 323, 1718-1722

42. Hollenstein, K. et al. (2007) Structure and mechanism of ABC transporter proteins. Curr. Opin. Struct. Biol. 17, 412-418

43. Jardetsky, O. (1966) Simple allosteric model for membrane pumps. Nature 211, 969-970

48. Burkhard, K.A. and Wilks, A. (2008) Functional characterization of the Shigella dysenteriae heme $\mathrm{ABC}$ transporter. Biochemistry 47, 7977-7979

44. Dawson, R.J. et al. (2007) Uptake or extrusion: crystal structures of full ABC transporters suggest a common mechanism. Mol. Microbiol. 65, 250-257

45. van der Does, C. and Tampé, R. (2004) How do ABC transporters drive transport? Biol. Chem. 385, 927-933

46. Higgins, C.F. and Linton, K.J. (2004) The ATP switch model for ABC transporters. Nat. Struct. Mol. Biol. 11, 918-926

47. Goetz, B.A. et al. (2009) Distinct gate conformations of the ABC transporter BtuCD revealed by electron spin resonance spectroscopy and chemical cross-linking. FEBS Lett. $583,266-270$ 
49. Oldham, M.L. et al. (2008) Structural insights into ABC transporter mechanism. Curr.

50. Zhang, Z. et al. (2003) A transporter of Escherichia coli specific for L- and D-methionine is the prototype for a new family within the ABC superfamily. Arch. Microbiol. 180, 88100

7

54. Dawson, R.J. and Locher, K.P. (2007) Structure of the multidrug ABC transporter Sav1866 from Staphylococcus aureus in complex with AMP-PNP. FEBS Lett. 581, 935938

55. Jones, P.M. and George, A.M. (2005) Multidrug resistance in parasites: ABC transporters, P-glycoproteins and molecular modelling. Int. J. Parasitol. 35, 555-566 
1 56. Dong, J. et al. (2005) Structural basis of energy transduction in the transport cycle of $2 \quad$ MsbA. Science 308, 1023-1028

3

4 57. Rothnie, A. et al. (2005) The coupling mechanism of P-glycoprotein involves residue L339 in the sixth membrane spanning segment. FEBS Lett. 579, 3984-3990

6

7 58. Zolnerciks, J.K. et al. (2007) Evidence for a Sav1866-like architecture for the human multidrug transporter P-glycoprotein. FASEB J. 21, 3937-3948

59. Serohijos, A.W. et al. (2008) Diminished self-chaperoning activity of the DeltaF508 mutant of CFTR results in protein misfolding. PLoS Comput. Biol. 4, e1000008

60. Oancea, G. et al. (2009) Structural arrangement of the transmission interface in the ABC

62. O'Mara, M.L. and Tieleman, D.P. (2007). P-glycoprotein models of the apo and ATPbound states based on homology with Sav1866 and MalK. FEBS Lett. 581, 4217-4222

61. Pagant, S. et al. (2008) Mapping of interdomain interfaces required for the functional architecture of Yor1p, a eukaryotic ATP-binding cassette (ABC) transporter. J. Biol. Chem. 283, 26444-26451 
63. Ravna, A.W. et al. (2007) Molecular model of the outward facing state of the Pglycoprotein (ABCB1), and comparison to a model of the human MRP5 (ABCC5). Theor. Biol. Med. Model. 4, 33-46

64. McDevitt, C.A. et al. (2008) Structural insights into P-glycoprotein (ABCB1) by small angle X-ray scattering and electron crystallography. FEBS Lett. 582, 2950-2956

65. Ward, A. et al. (2009) Nucleotide dependent packing differences in helical crystals of the ABC transporter MsbA. J. Struct. Biol. 165, 169-175

66. Rosenberg, M.F. et al. (1997) Structure of the multidrug resistance P-glycoprotein to 2.5 $\mathrm{nm}$ resolution determined by electron microscopy and image analysis. J. Biol. Chem. 272 , 10685-10694

67. Rosenberg, M.F. et al. (2001) Repacking of the transmembrane domains of P-glycoprotein during the transport ATPase cycle. EMBO J. 20, 5615-5625

68. Rosenberg, M.F. et al. (2003) Three-dimensional structures of the mammalian multidrug resistance P-glycoprotein demonstrate major conformational changes in the transmembrane domains upon nucleotide binding. J. Biol. Chem. 278, 8294-8299

69. Rosenberg, M.F. et al. (2005) Three-dimensional structure of P-glycoprotein: the transmembrane regions adopt an asymmetric configuration in the nucleotide-bound state. $J$ Biol Chem. 280, 2857-2862 
70. Lee, J.Y. et al. (2008) Nucleotide-induced structural changes in P-glycoprotein observed by electron microscopy. J. Biol. Chem. 283, 5769-5779

71. Velarde, G. et al. (2001) Three-dimensional structure of transporter associated with antigen processing (TAP) obtained by single Particle image analysis. J. Biol. Chem. 276, 46054-46063

7

8 72. Higgins, C.F. and Gottesman, M.M. (1992) Is the multidrug transporter a flippase? Trends Biochem. Sci. 1, 18-21

73. Tam, R. and Saier, M.H. (1993) A bacterial periplasmic receptor homologue with catalytic activity: cyclohexadienyl dehydratase of Pseudomonas aeruginosa is homologous to receptors specific for polar amino acids. Res. Microbiol. 144, 165-169

74. Saurin, W. and Dassa, E. (1994) Sequence relationships between integral inner membrane proteins of binding protein-dependent transport systems: evolution by recurrent gene duplications. Protein Sci. 3, 325-344

75. Saurin, W. et al. (1999) Getting in or out: early segregation between importers and exporters in the evolution of ATP-binding cassette (ABC) transporters. J. Mol. Evol. 48, $22-41$

76. Senior, A.E. et al. (1995) The catalytic cycle of P-glycoprotein. FEBS Lett. 377, 285-289

77. Humphrey, W. et al. (1996) VMD: visual molecular dynamics. J. Mol. Graph. 14, 33-38 
78. Sharma, S. and Davidson, A.L. (2000) Vanadate-induced trapping of nucleotide by the purified maltose transport complex requires ATP hydrolysis. J. Bacteriol. 182, 6570-6576

79. Westfahl, K.M. et al. (2008) Functionally important ATP binding and hydrolysis sites in Escherichia coli MsbA. Biochemistry 47, 13878-13886

80. Vergani, P. et al. (2005) CFTR channel opening by ATP-driven tight dimerization of its nucleotide-binding domains. Nature 433, 876-880

81. Oswald, C. et al. (2006) The motor domains of ABC-transporters. What can structures tell us? Naunyn Schmiedebergs Arch. Pharmacol. 372, 385-399

82. Higgins, C.F. (2007) Multiple molecular mechanisms for multidrug transporters. Nature $446,749-757$

83. Tombline, G. and Senior, A.E. (2005) The occluded nucleotide conformation of Pglycoprotein. J. Bioenerg. Biomembr. 37, 497-500

84. Sauna, Z.E. et al. (2007) Catalytic cycle of ATP hydrolysis by P-Glycoprotein: evidence for formation of the E.S reaction intermediate with ATP- $\gamma$-S, a nonhydrolyzable analogue of ATP. Biochemistry 46, 13787-13799

85. Jones, P.M. and George, A.M. (2007) Nucleotide-dependent allostery within the ABC transporter ATP-binding cassette. J. Biol. Chem. 282, 22793-22803 
86. Jones, P.M. and George, A.M. (2009) Opening of the ADP-bound active site in the ABC transporter ATPase dimer: Evidence for a constant contact, alternating sites model for the catalytic cycle. Proteins $75,387-396$

87. Buchaklian, A.H. and Klug, C.S. (2005) Characterization of the Walker A motif of MsbA using site-directed spin labeling electron paramagnetic resonance spectroscopy. Biochemistry 44, 5503-5509

88. Buchaklian, A.H. and Klug, C.S. (2006) Characterization of the LSGGQ and H motifs from the Escherichia coli lipid A transporter MsbA. Biochemistry 45, 12539-12546

89. Qu, Q. et al. (2003) Stoichiometry and affinity of nucleotide binding to P-glycoprotein during the catalytic cycle. Biochemistry 42, 1170-1177

90. Grote, M. et al. (2008) A comparative electron paramagnetic resonance study of the nucleotide-binding domains' catalytic cycle in the assembled maltose ATP-binding cassette importer. Biophys. J. 95, 2924-2938

91. van Veen, H.W. et al. (2000) The homodimeric ATP-binding cassette transporter LmrA mediates multidrug transport by an alternating two-site (two-cylinder engine) mechanism. EMBO J 19, 2503-2514

92. Guex, N. and Peitsch, M.C. (1997) SWISS-MODEL and the Swiss-PdbViewer: An environment for comparative protein modeling. Electrophoresis 18, 2714-2723 


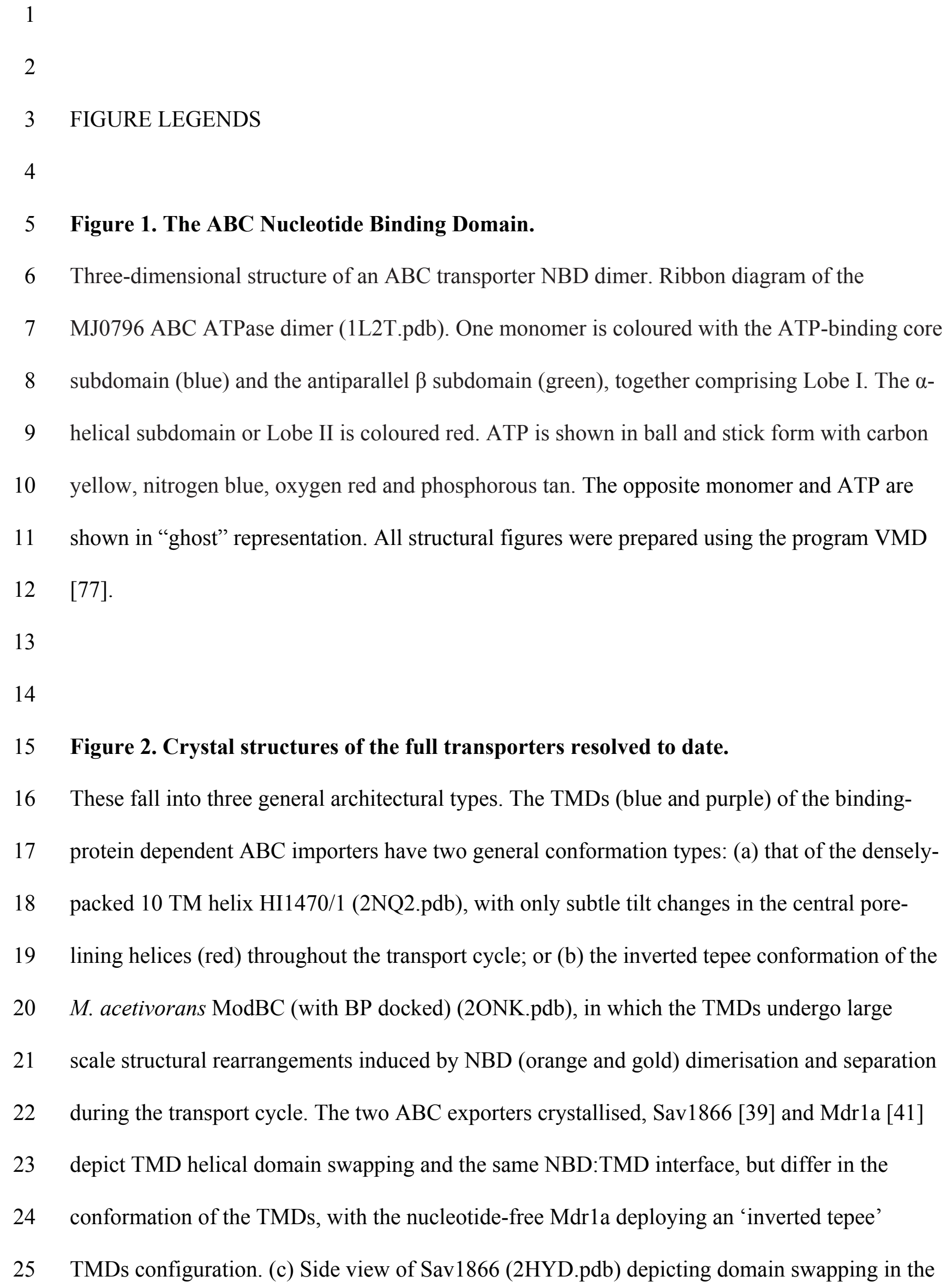


11 TMDs, which form divergent open wings in the nucleotide-bound state. (d) Front view of

2 Sav1866 depicting the two coupling helices (short, almost horizontal purple and blue cylinders

3 just above the NBDs).

4

5

6 Figure 3. Simple two-state scheme for ABC importers.

7 In the absence of substrate, the transporter is in a conformation in which the NBD dimer

8 interface is open and the translocation pathway is exposed only to the cytoplasm (left panel).

9 Interaction of substrate-bound BP with the closed extracellular side of the TMDs in the

10 presence of ATP, triggers a global conformational change in which the NBDs close to promote

11 ATP hydrolysis, substrate-bound BP becomes tightly bound to the TMDs, and both BP and

12 TMDs open at the periplasmic surface of the membrane to facilitate the transfer of substrate

13 from the BP to a binding site in the membrane (centre panel). Following ATP hydrolysis,

14 which destabilizes the NBD dimer, the transporter returns to the inward facing state and the

15 substrate completes its translocation across the membrane (right panel).

16

Figure 4. The densely packed TMDs of BtuCD and HI1470/71.

These $\mathrm{ABC}$ transporters show relatively subtle conformational changes between the nucleotide-

free and nucleotide-bound states, with the major structural rearrangements occurring as a pivoting motion in TM5 and its short extramembrane extension, TM5a (coloured red in TMD1 and blue in TMD2). (a) In the apo conformation of BtuCD (1L79.pdb), the outwards facing conformation is characterised by a narrowing of the distance between the two TM5s, closing the TMD to the intracellular side. (b) The crystal structure of BtuCD in complex with its binding protein (2QI9.pdb) shows an intermediate conformation of TM5 and TM5a. (c) The 
nucleotide bound conformation of HI1470/1 (2NQ2.pdb) shows the two TM5s spayed apart towards the cytosolic side of the membrane, with the helices parallel and the cavity slightly open to the cytoplasm.

4 5

Figure 5. The Transmission Interface.

Interaction of the coupling helices $(\mathrm{CH})$ of the TMD intracytoplasmic loops with the NBD. Ribbon diagram of the Sav1866 NBD (2ONJ.pdb) with Lobe I yellow, Lobe II green and the Q-loop purple. Key structural elements discussed in the text are labelled. The ATP analogue AMP-PNP is shown in ball and stick form with carbon grey, nitrogen blue, oxygen red and phosphorous tan. The Sav1866 CHs are shown in $\mathrm{C} \alpha$ trace with $\mathrm{CH} 1$ blue and $\mathrm{CH} 2$ red. The NBDs from six whole ABC importer structures were superimposed on the Sav1866 NBD using the "best fit with structural alignment" option in SwissPDBviewer [92]. The CHs of the importers are shown in $\mathrm{C} \alpha$ trace with BtuCD (1L7V.pdb) grey, BtuCD-F (2QI9.pdb) orange, HI1470/1 (2NQ2.pdb) cyan, MalG (2R6G.pdb) steel blue, MetN (3DHW.pdb) tan and ModA (2ONK.pdb) pink.

Figure 6. Scheme for the catalytic cycle of the NBD dimer.

The three current models for ATP binding and hydrolysis. NBDs are depicted as blue and orange semi-circular blocks, with the dimer closed, fully open, or partly opened, as rendered in each model. TMDs are not shown.

(a) Switch model [45] and [46]. Step I: in the resting state the nucleotide-free NBDs are in an open dimer configuration. ATP binds cooperatively to the two active sites. Step II: binding of 
two ATPs drives the formation of the closed NBD dimer. In some transporters ATP binding to

2 the two sites is proposed to be a stochastic process while in others one site has higher affinity

3 for nucleotide. Step III: the two ATP molecules are hydrolysed sequentially with the hydrolysis

4 products remaining bound to the protein. Step IV: sequential release of Pi and then ADP

5 restores the transporter to its basal configuration [46].

6

7

(b) Alternating sites model [76]. The catalytic pathway is shown in a number of intermediate states I-VI. Initial loose binding of ATP at both sites triggers formation of the closed NBD dimer (States I => II => III). The occluded ATP conformation occurs at state III, with only one ATP bound tightly and committed to hydrolysis. This ATP (bold type in State III) enters the transition state and forms Pi and ADP (III => IV). Pi and ADP are released as the dimer opens (V, VI), and binding of new ATP would occur. Thus, in contrast to the switch model (Figure 6a), hydrolysis of one ATP is sufficient to drive the NBD dimer to the fully open state, product release and rebinding of a new ATP molecule. The transporter is proposed to retain a "memory" of which site last hydrolysed ATP such that ATP is subsequently hydrolysed in the opposite site resulting in alternating ATP hydrolysis [76] and [81].

(c) Constant-contact model [84], [85] and [86]. For each possible state of the NBD active site there are two distinct substates, either occluded (closed) or open (allowing nucleotide exchange), or in the case of the empty site, high or low affinity for nucleotide. Each active site cycles in the sequence: ATP-open, ATP-occluded, ADP(+Pi)-occluded, ADP-open, empty-low affinity, empty-high affinity. The two active sites function 180 degrees out of phase with respect to this cycle and ATP hydrolysis alternates between the opposite sites. The NBDs remain in contact throughout the catalytic cycle, with opening and closing of the active sites occurring by way of intrasubunit conformational changes within the NBD monomers [85]. Step 
1 2

I: the ATP-bound active site is closed and with opposite site empty. ATP hydrolysis occurs.

2 Step II: with hydrolysis products still bound in the occluded active site, the empty site switches

3 to high affinity, enabling ATP binding. Step III: ATP binding to the empty site and Pi release

4 from the occluded post-hydrolysis site promotes opening of the ADP-bound site and closing of

5 the ATP-bound site. Step IV: ADP release from the open site enables ATP hydrolysis in the

6 opposite site as in Step I, completing the half cycle. 
Click here to download high resolution image

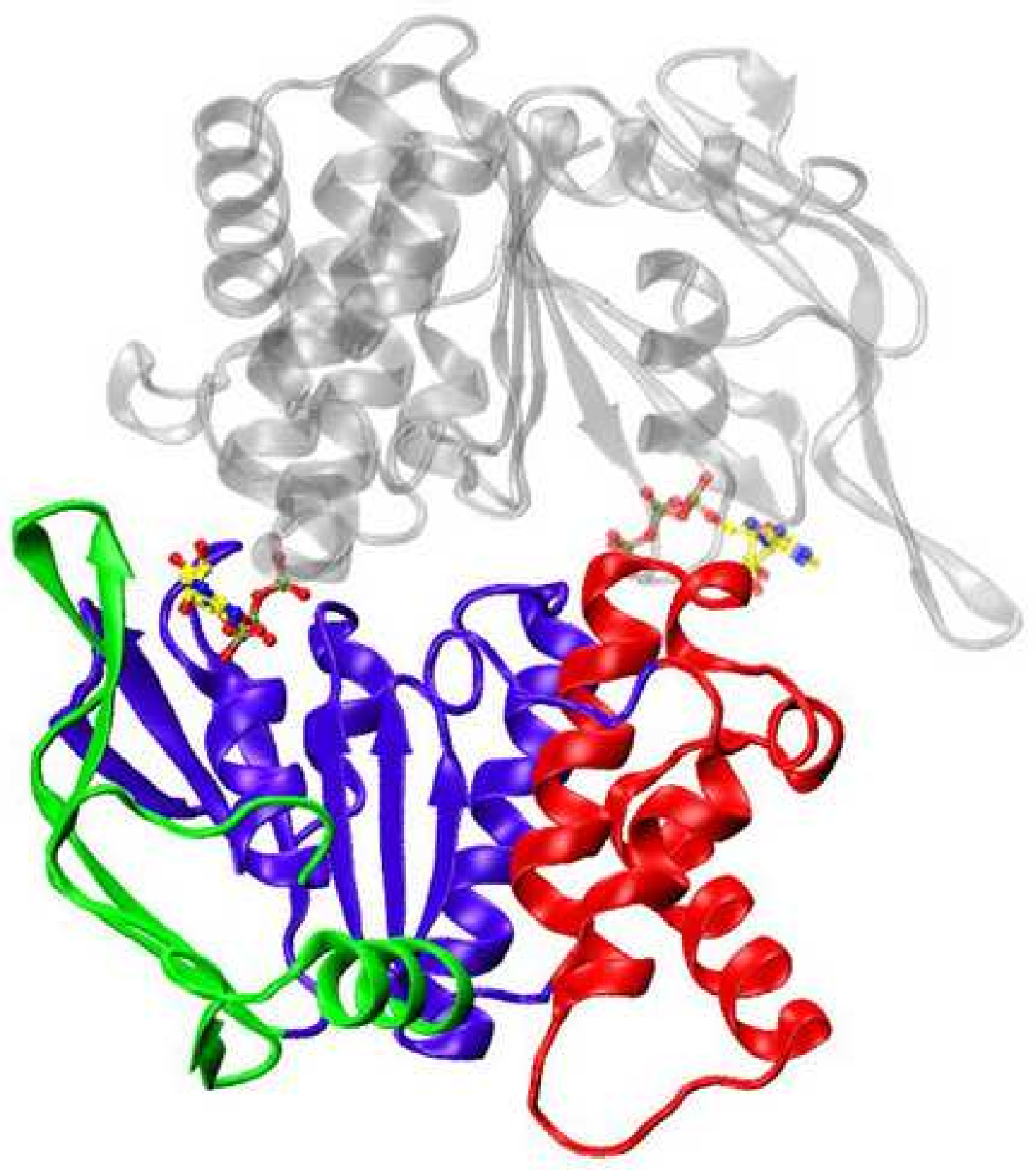


Click here to download high resolution image

(a)

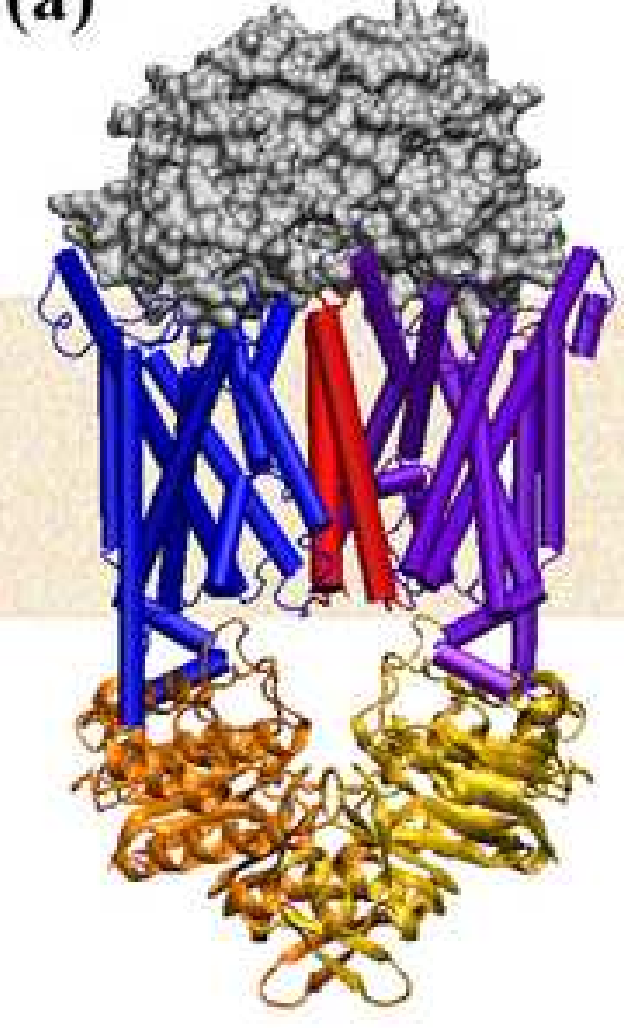

(b)

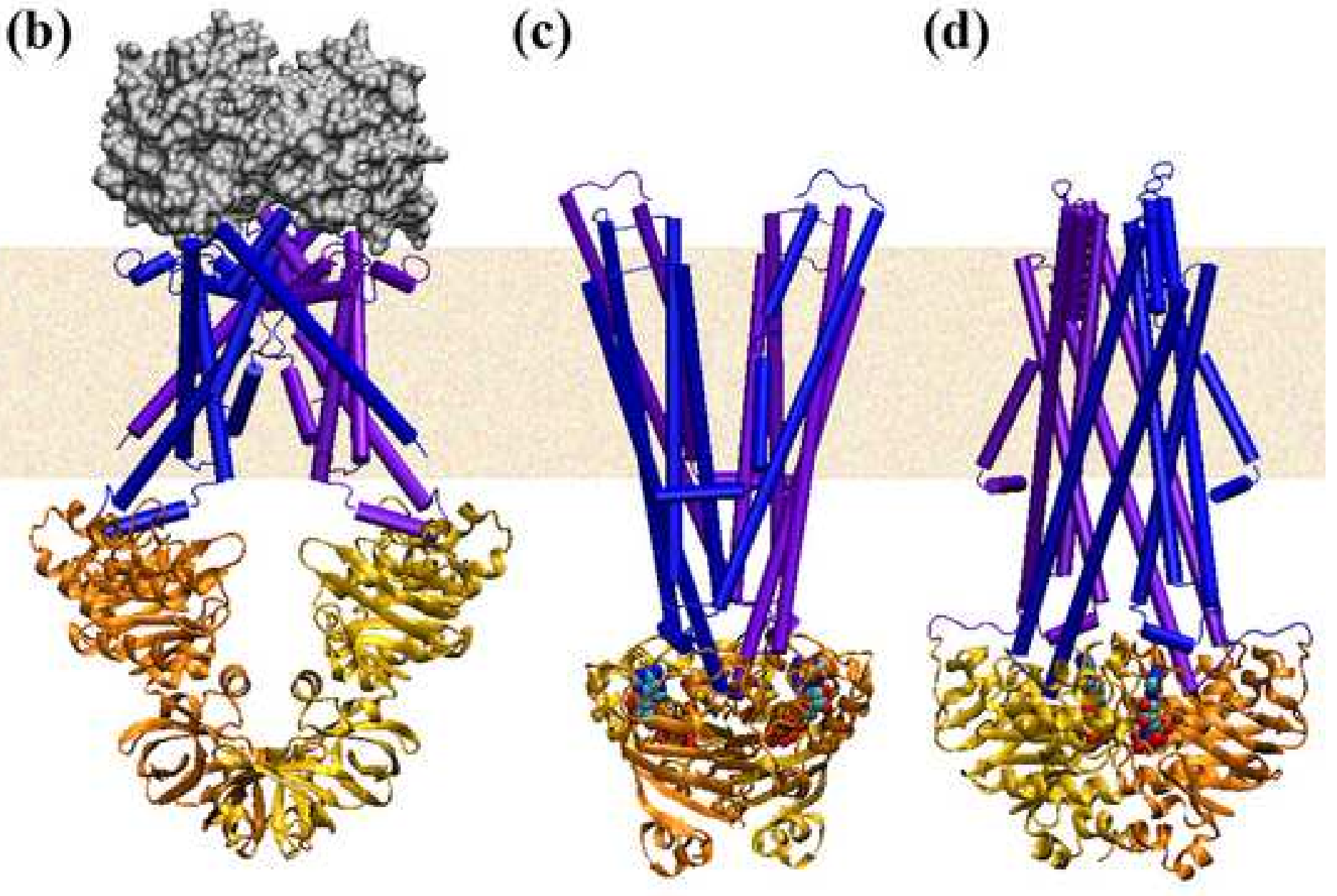

(c)

(d)

c)

$$
\text { d) }
$$

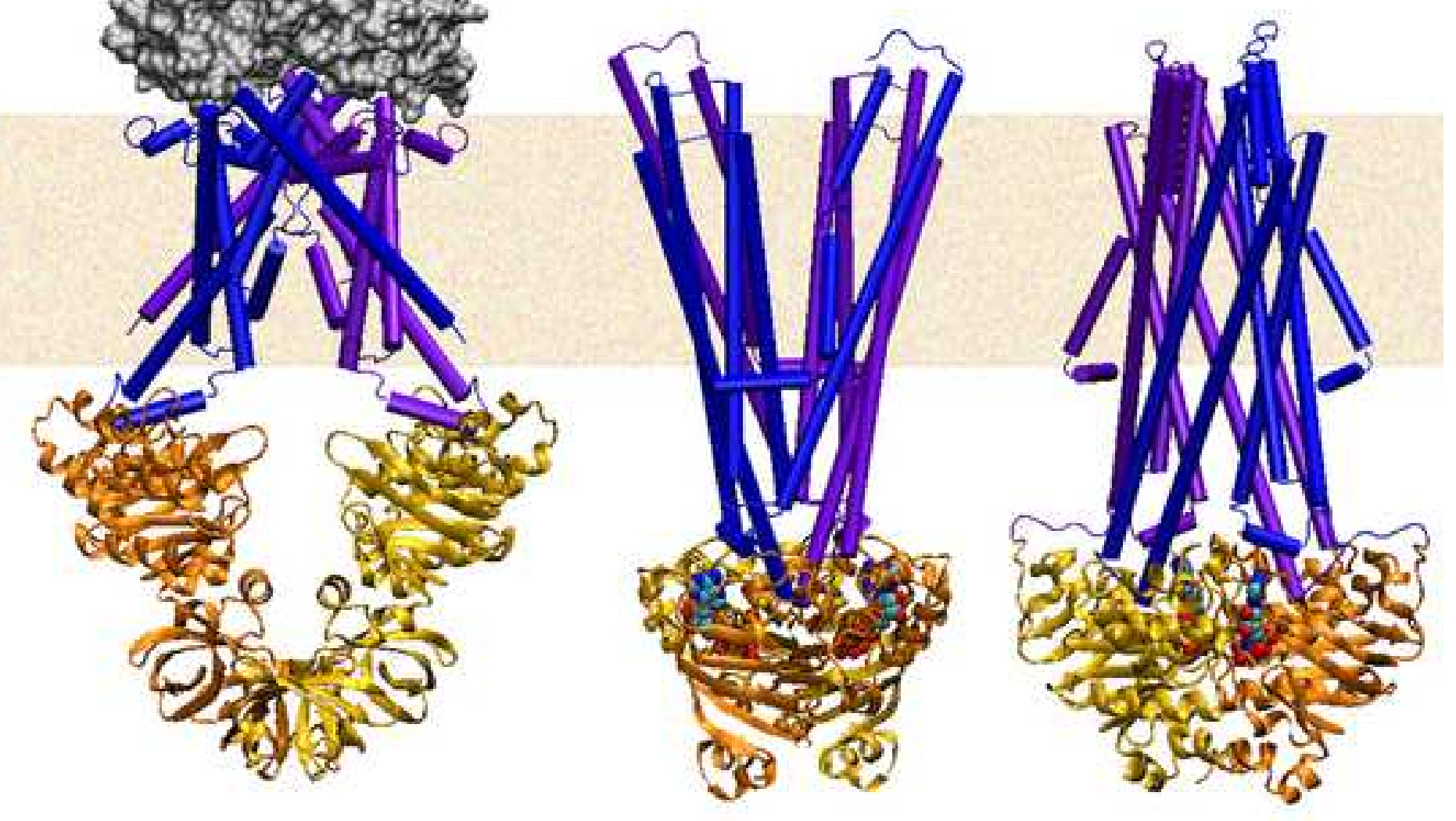




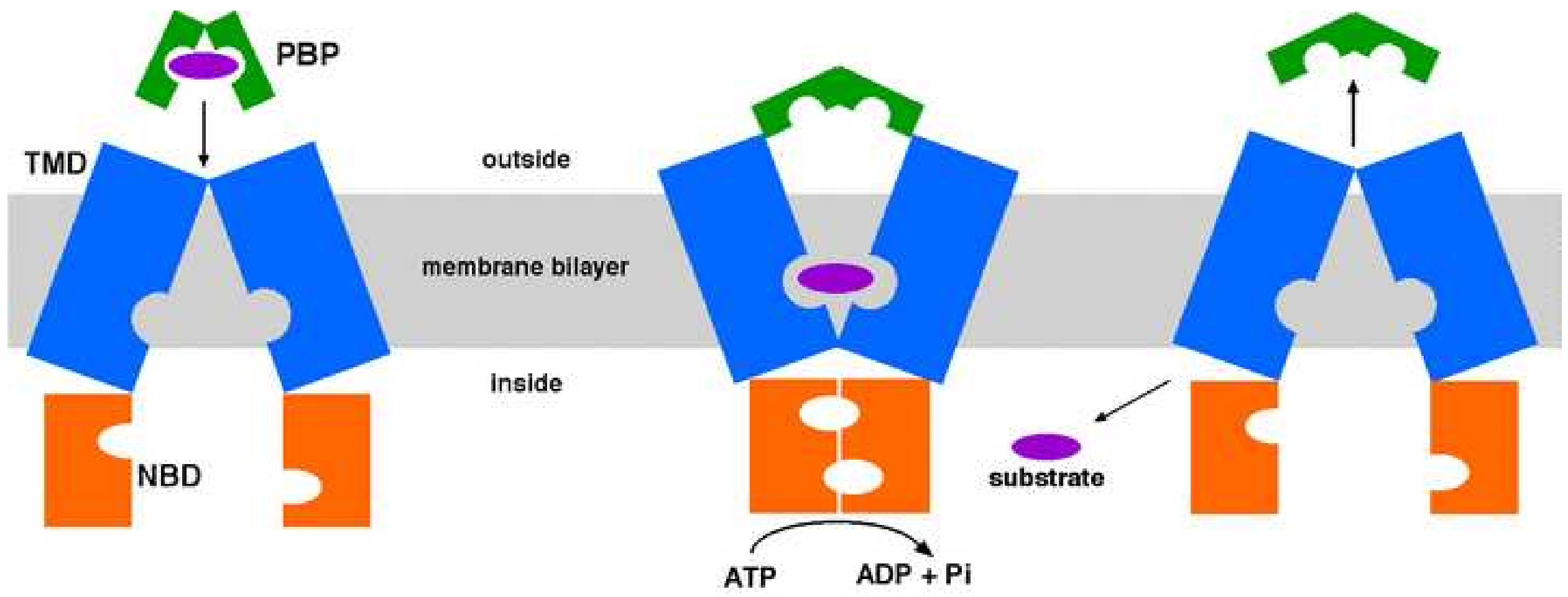


Click here to download high resolution image
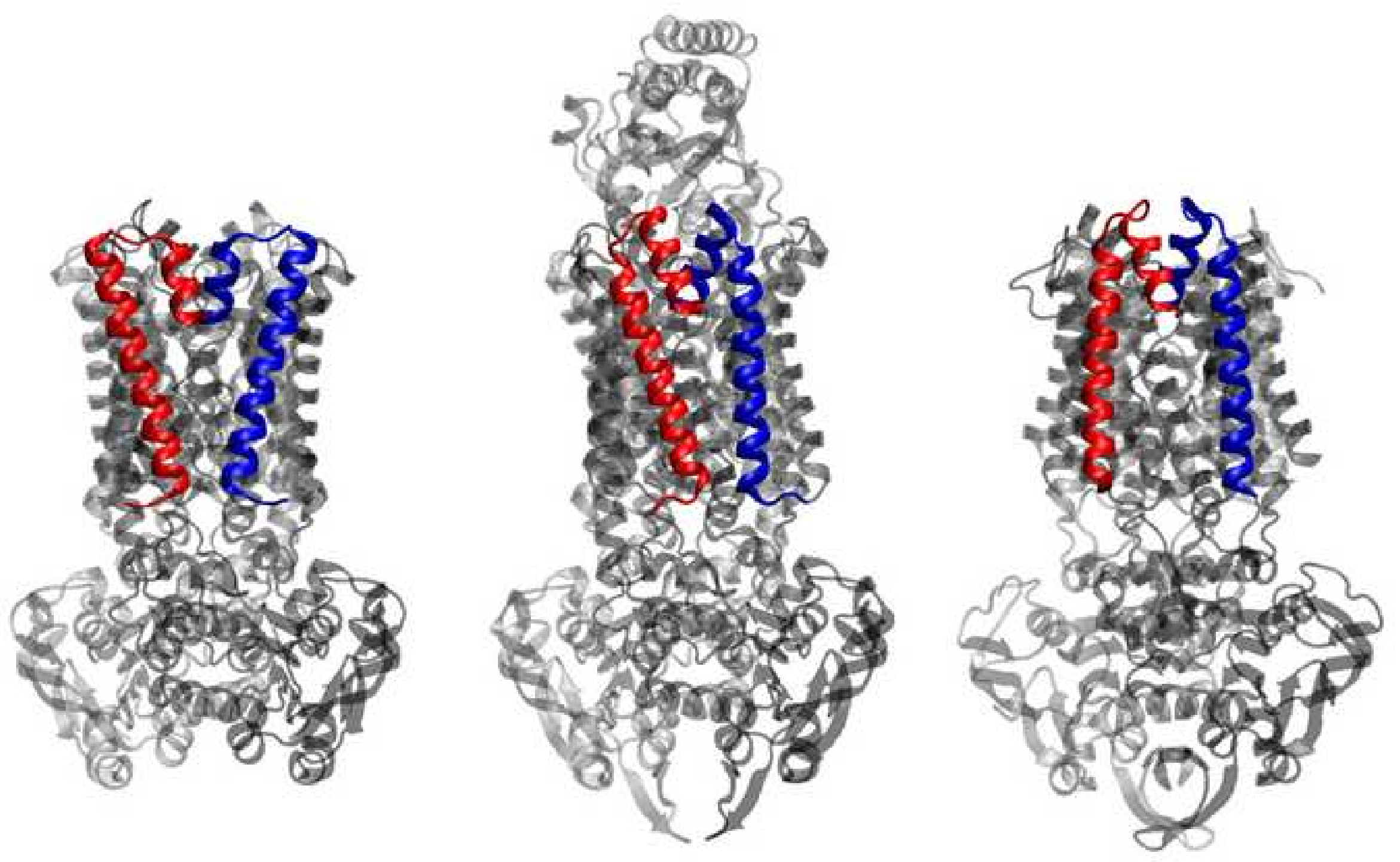


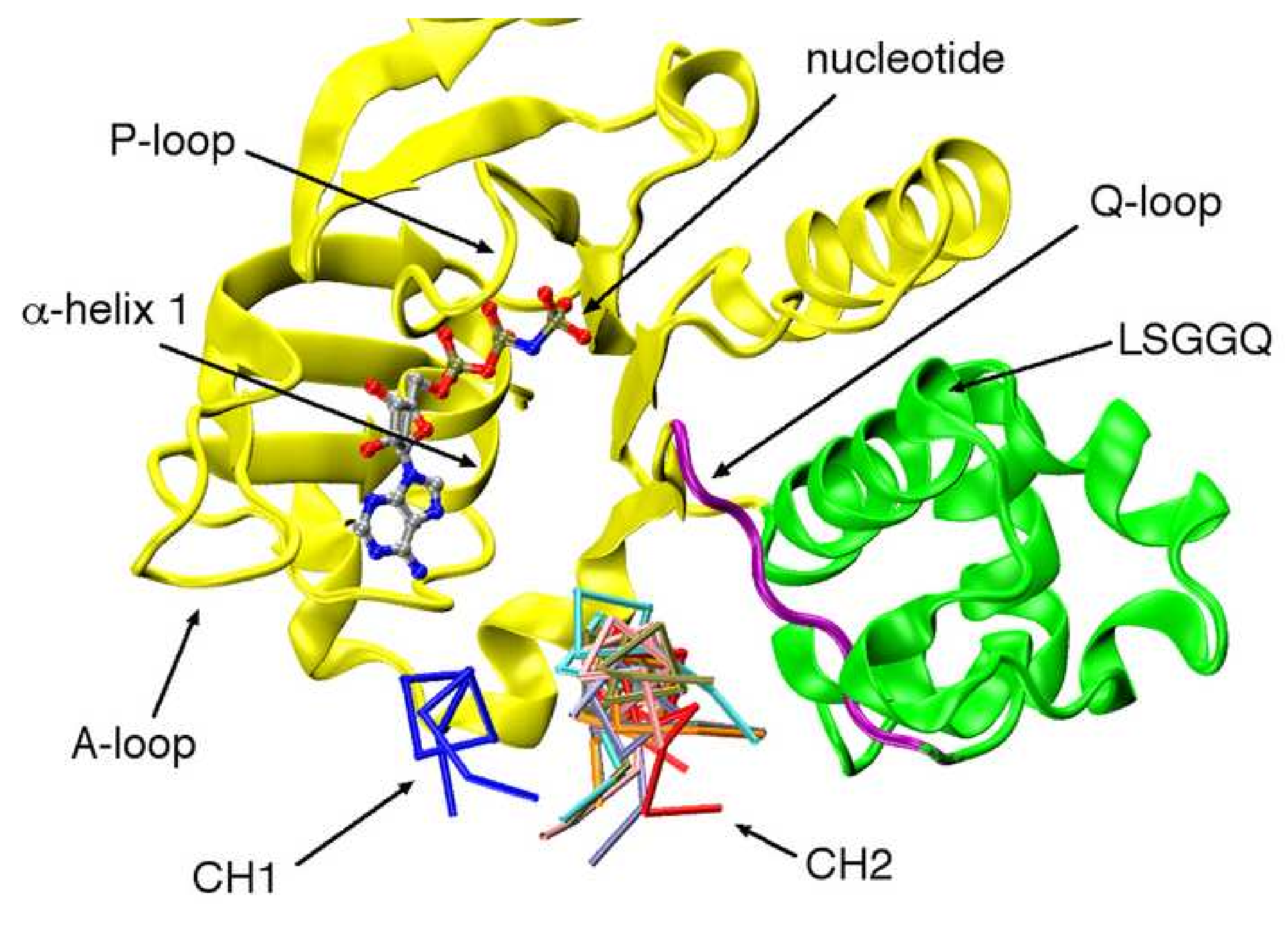

.

(a)
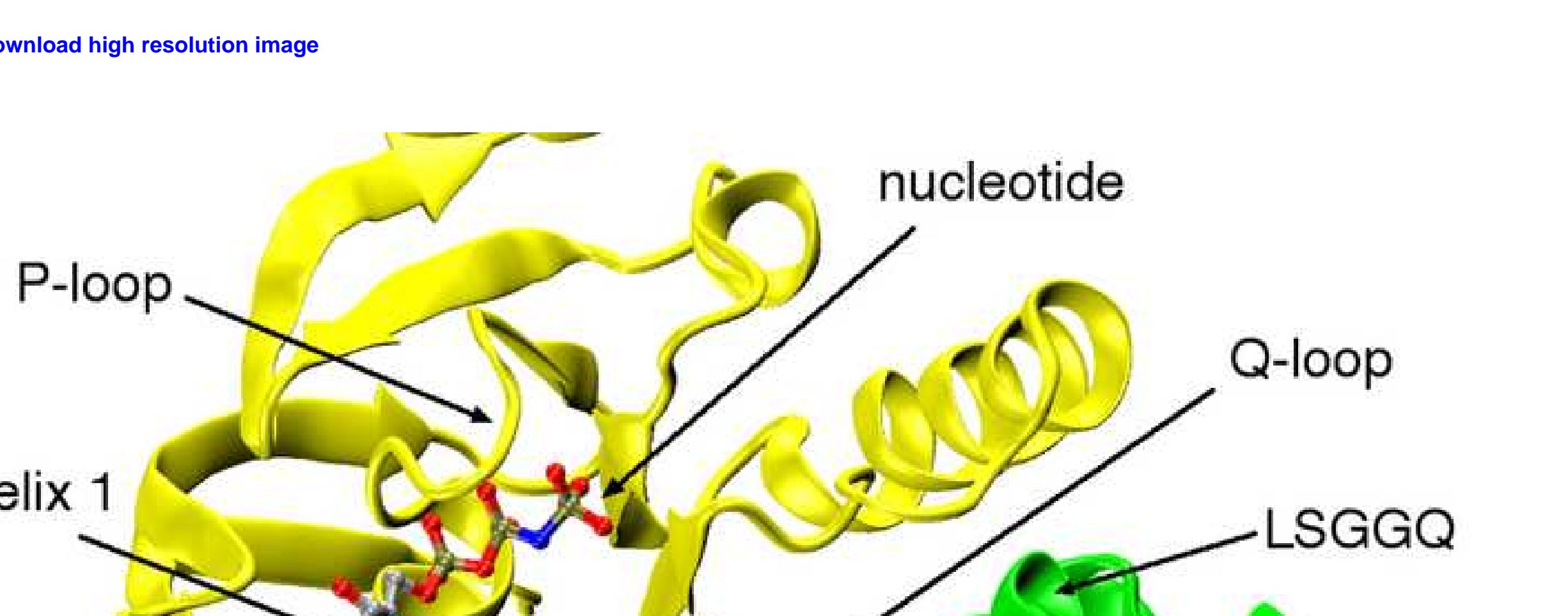
(a)
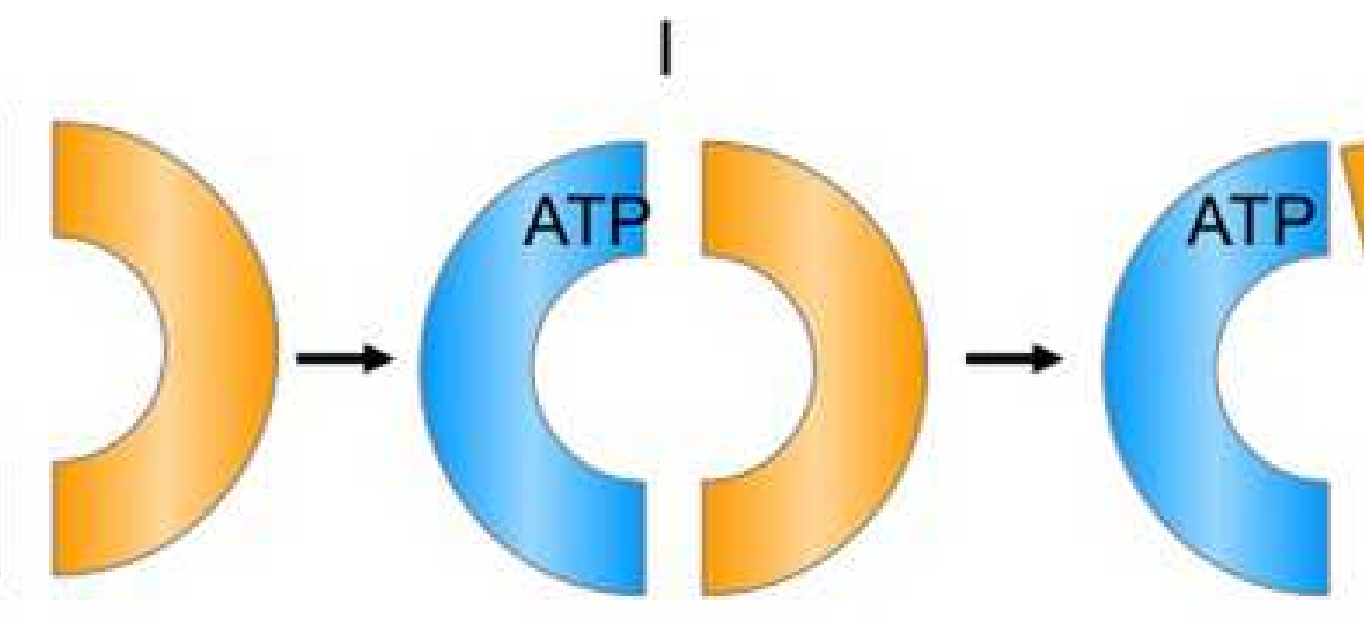

IV
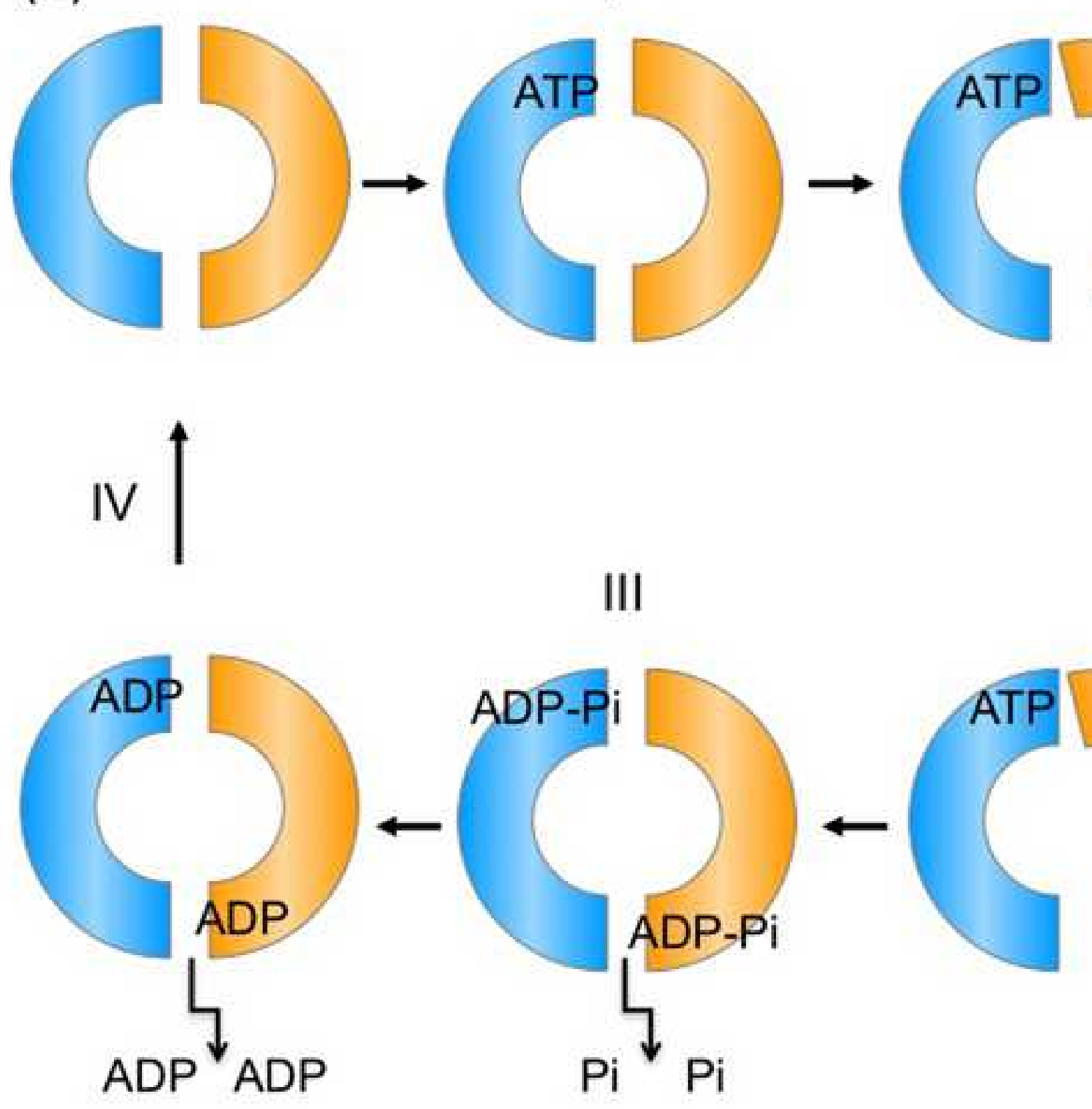

ATP

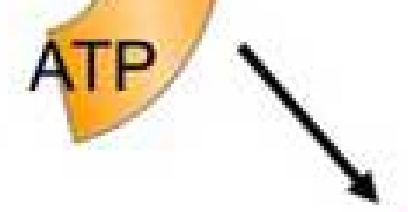

II

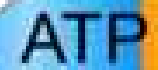

ADP-Pi

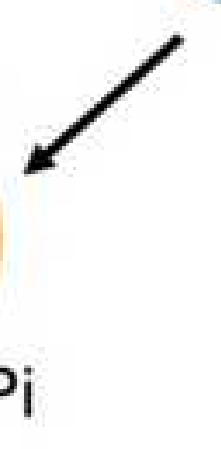


(b)

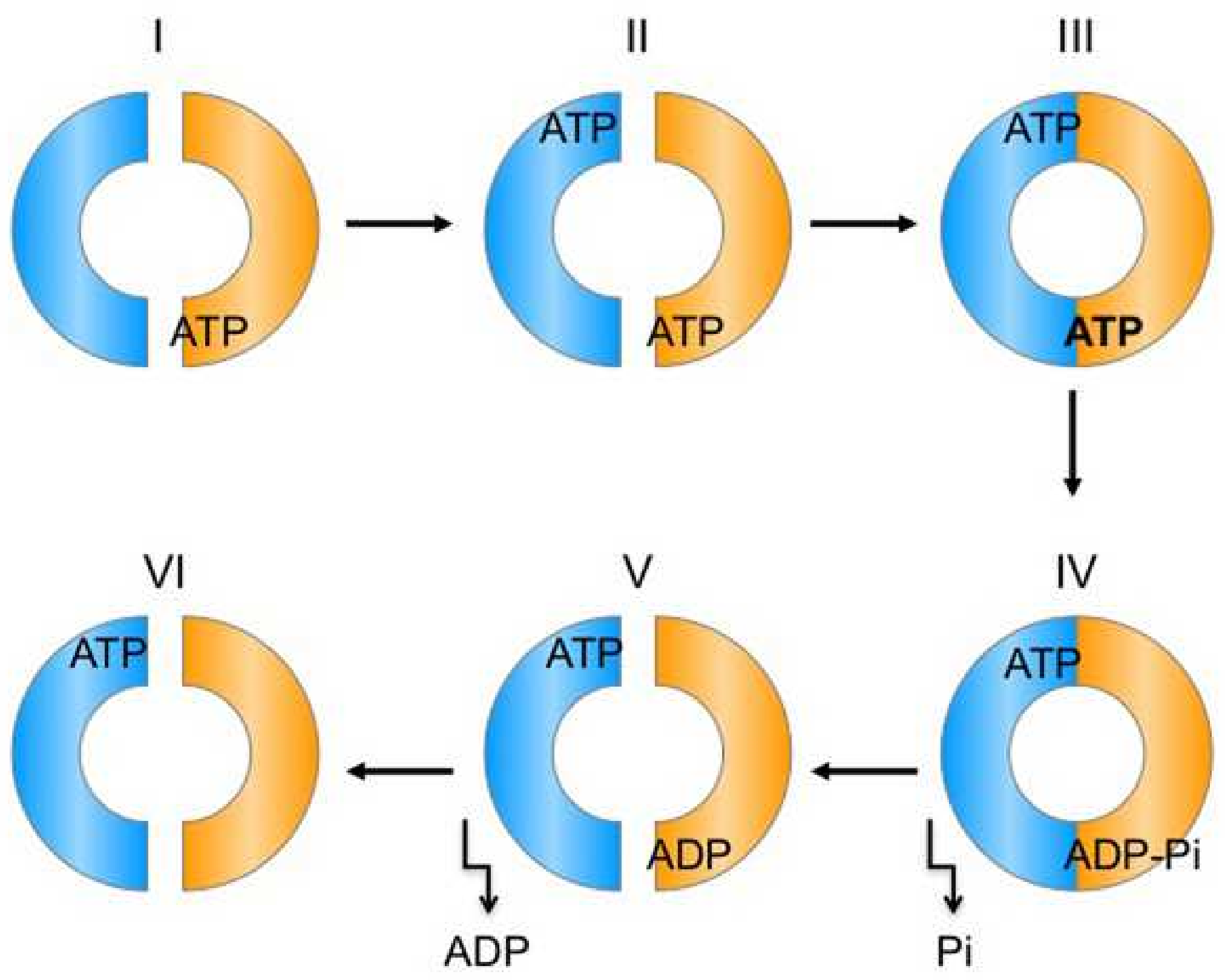


(c)
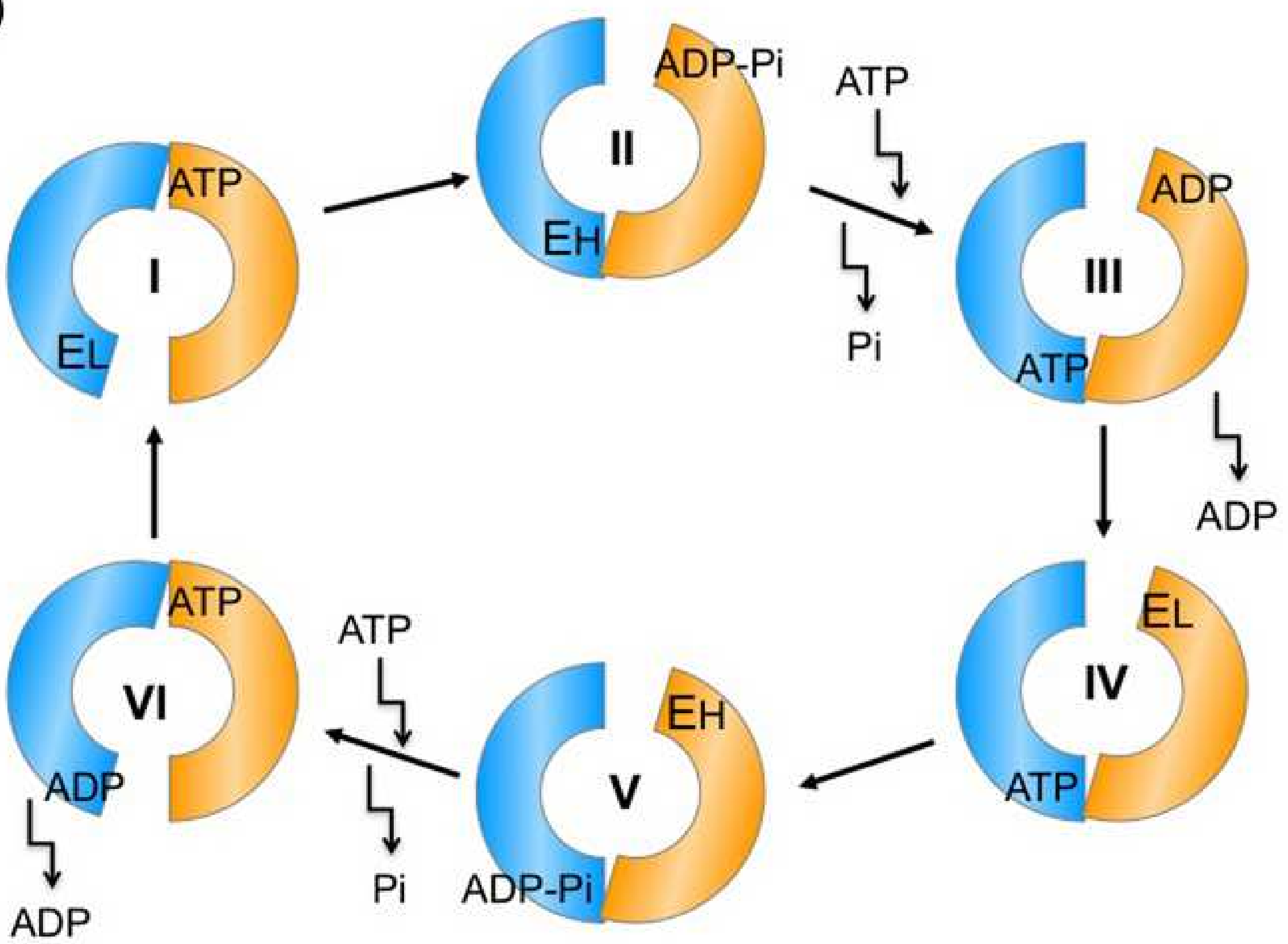